\title{
PROBLÈMES \\ DE STABILITÉ RELATIFS \\ AUX BARRAGES A CONTREFORTS \\ ET PLUS PARTICULIÈREMENT \\ AUX BARRAGES A VOUTES \\ MULTIPLES
}

(suite et fin) *

\section{PAR \\ P. PATIN * * \\ ET}

G. DEGEORGES ** *

DEUXIĖME PARTIE

\section{Stabilité élastique d'un ensemble constitué par un contrefort et les deux voûtes adjacentes}

Alors que, jusqu'ici, nous avons regardé la rigidité transversale du contrefort comme étant suffisamment grande pour que celui-ci ne puisse flamber, nous examinerons maintenant le cas plus général où le phénomène de déversement s'accompagne d'un voilement du contrefort.

Nous supposerons implicitement que la stabilité élastique propre des voûtes n'est pas mise en cause au cours des déformations envisagées. On sait que pour les élancements usuels et les contraintes actuellement admises, le coefficient de sécurité intrinsèque des ares est généralement confortable. Toutefois, associé à celui des contreforts, le flambement des arcs constitue un problème complexe, mais, comme les voûtes et les contreforts ne présenteront pour ainsi dire jamais le même coefficient de sécurité vis-à-vis du flambement, nous poserons que celui des contreforts est le plus faible, donc que tout phénomène de voilement affectera en priorité les contreforts.

\section{A - HYpothises :}

Outre les hypothèses de la première partie, qui sont conservées, nous supposerons cette fois que la plaque qui constitue le contrefort est un triangle

* Voir La Houille Blanche, n" 7-1965, pp. 681 à 690.

* Directeur Général de la Compagnie d'Ingénieurs et Techniciens d'Etudes, Paris.

* * Ingénieur-Conseil à la Compagnie d'Ingénieurs et Techniciens d'Etudes, Paris. isocèle dont les côtés égaux sont les faces amont et aval de ce contrefort (fig. 10).

En pratique, la somme des fruits varie entre 1 el 1,2, mais le fruit du parement amont est presque toujours supérieur à celui du parement aval.

On emploiera les procédés de la résistance des matériaux en faisant appel à la thérie des poutres et non à celle des plaques.

Le mode de déformation du contrefort en dehors de son plan moyen hors charge, sera regardé comme résultant de la superposition d'une torsion $\varphi=\varphi(x)$ autour de l'axe de symétrie, c'est-à-dire autour de la verticale, et d'une flexion latérale le long de cet axe définie par la fonction $z=f(x)$. Bien qu'un tel choix puisse paraitre arbitraire, il nous paraît englober les différents modes de déformation possibles.

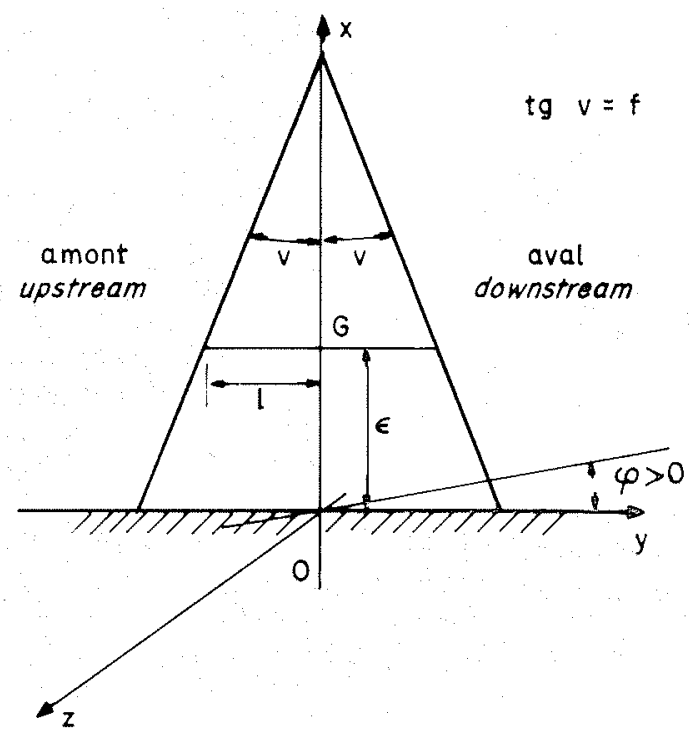

$10 /$ 


\section{P. PATIN et G. DEGEORGES}

\section{B - Bases THÉoRIQUeS DE L'ÉTUdE :}

Nous ferons à nouveau appel à la méthode de l'énergie, qui revient, nous l'avons rappelé, à établir les conditions de minimum de la fonction :

$$
(\mathrm{U}-\mathrm{T}) \quad \text { c'est-à-dire } \delta(\mathrm{U}-\mathrm{T})=0
$$

Mais alors que précédemment le phénomène était complètement défini par la connaissance exclusive des paramètres $a$, nous ignorons ici la forme analytique des fonctions $\varphi$ et $f$ qui caractérisent le voilement.

Faire choix a priori de fonctions simplement «ajustées», c'est-à-dire satisfaisant aux conditions aux limites, revient à introduire des forces complémentaires fictives ramenant la déformée réelle en coïncidence avec la déformée supposée.

On raidit ainsi le système et on aboutit à une valeur trop grande de la charge critique.

La méthode de Ritz fait appel au développement en série des fonctions $\varphi$ et $f$ de la forme :

$$
\begin{gathered}
z=a_{0}+a_{1} f_{1}+a_{2} f_{2}+a_{3} f_{3}+\ldots \\
\varphi=b_{0}+b_{1} \varphi_{1}+b_{2} \varphi_{2}+b_{3} \varphi_{3}+\ldots
\end{gathered}
$$

Les séries de Fourier apparaissent comme un cas particulier de cette forme de développement.

Les séries ci-dessus doivent bien entendu être convergentes dans l'intervalle considéré, et toutes les fonctions du développement satisfont séparément aux conditions aux limites.

L'expression $(\mathrm{U}-\mathrm{T})$ peut se mettre sous la forme :

$$
(\mathrm{U}-\mathrm{T})=\mathrm{F}\left(a_{0}, a_{1}, a_{2} \ldots ; b_{0}, b_{1}, b_{2} \ldots\right)
$$

$$
\text { et : } \begin{aligned}
\delta(\mathrm{U}-\mathrm{T}) & =\frac{\delta \mathrm{F}}{\delta a_{0}} d a_{0}+\frac{\delta \mathrm{F}}{\delta a_{1}} d a_{1}+\ldots \\
& +\frac{\delta \mathrm{F}}{\delta b_{0}} d b_{0}+\frac{\delta \mathrm{F}}{\delta b_{1}} d b_{1}+\ldots=0
\end{aligned}
$$

Les accroissements $d a_{0}, d a_{1} \ldots d b_{0}, d b_{1} \ldots$ étant arbitraires, on devra avoir simultanément :

$$
\begin{array}{ll}
\frac{\delta \mathrm{F}}{\delta a_{0}}=0 ; & \frac{\delta \mathrm{F}}{\delta a_{1}}=0 ; \quad \ldots \\
\frac{\delta \mathrm{F}}{\delta b_{0}}=0 ; & \frac{\delta \mathrm{F}}{\delta b_{1}}=0 ; \quad \ldots
\end{array}
$$

Puisque nous avons convenu de négliger les termes de degré supérieur au second dans le calcul de (U - T), F sera une fonction quadratique des paramètres $a_{0}, a_{1} \ldots b_{0}, b_{1}$.

Les dérivées partielles ci-dessus seront donc du premier degré par rapport à ces mêmes paramètres.

Le système linéaire homogène ainsi obtenu ne pourra avoir de solution différente de 0 qu'à la condition que les déterminants formés par les coefficients des inconnues s'annulent.

On sera donc conduit à un processus de calcul analogue à celui du chapitre précédent.

\section{Remarque I :}

La condition de nullité du déterminant étant effectivement vérifiée, le système linéaire sera alors indéterminé. En d'autres termes, à une valeur arbitraire attribuée à l'un quelconque des paramètres, correspondra une valeur unique et bien définie de chacun des autres paramètres.

Ce caractère d'indétermination du système élastique déformé, n'est dû en fait qu'à l'artifice auquel nous avons eu recours pour être en mesure d'aborder le problème, lorsqu'on a décidé de négliger les termes de degré supérieur au second. On retrouve la même indétermination dans les études du flambement des poutres droites, lorsque l'on fait abstraction dans l'expression du rayon de courbure, figurant dans l'équation différentielle de déformation, du carré de la dérivée première en écrivant:

$$
\frac{1}{\rho}=\frac{d^{2} y / d x^{2}}{\left[1+(d y / d x)^{2}\right]^{3 / 2}} \simeq \frac{d^{2} y}{d x^{2}}
$$

La déformée n'est connue qu'à un facteur près. Mais si on prend la valeur exacte du rayon de courbure, lindétermination disparaît.

\section{Remarque II :}

Dans leur principe, les calculs qui découlent de l'application de la méthode de Ritz sont simples. Cependant, sur le plan pratique, on sera rapidement limité par leur ampleur qui s'accroît très vite avec le nombre de termes conservés dans les séries. Toutefois, l'expérience du procédé fait apparaître une convergence telle du résultat que le premier terme est souvent suffisant dans la plupart des cas.

Dans les calculs qui suivent, on évaluera en fonction des divers paramètres le travail fourni par les forces appliquées à l'élément et que nous avons précédemment analysées et la variation du potentiel interne du système.

Nous avons considéré les forces d'origine hydrostatique comme s'exerçant le long de la face amont du contrefort. Il faut donc définir le vecteur représentatif du déplacement d'un point quelconque de cette face.

Ce sera l'objet du paragraphe ci-dessous.

La variation totale du potentiel interne du système sera maintenant égale à la somme de celle des voûtes et de celle du contrefort.

Ce calcul fera l'objet du second paragraphe.

\section{C - Calcul des composantes du déplacement d'un POINT SITUÉ SUR LA FACE AMONT DU CONTRE- FORT :}

Soit le trièdre trirectangle de référence $O x y z$ dont le sens est précisé sur le croquis.

L'axe $O x$ coïncide avec l'axe de symétrie. Il est donc orienté suivant la verticale.

Il est aussi confondu avec la fibre moyenne avant déformation.

Le sens positif de rotation des sections horizontales, à la suite de la torsion est également indiqué sur le croquis.

\section{1'Déplacement d'un point $G$ de la fibre moyenne par rapport au trièdre Oxyz:}

La composante de ce déplacement suivant $\mathrm{O} z$ est donnée par la fonction $z=\mathrm{f}(x)$. Mais, du fait de la rotation des sections droites dues à la torsion, la flexion s'effectue dans un plan variable faisant un 

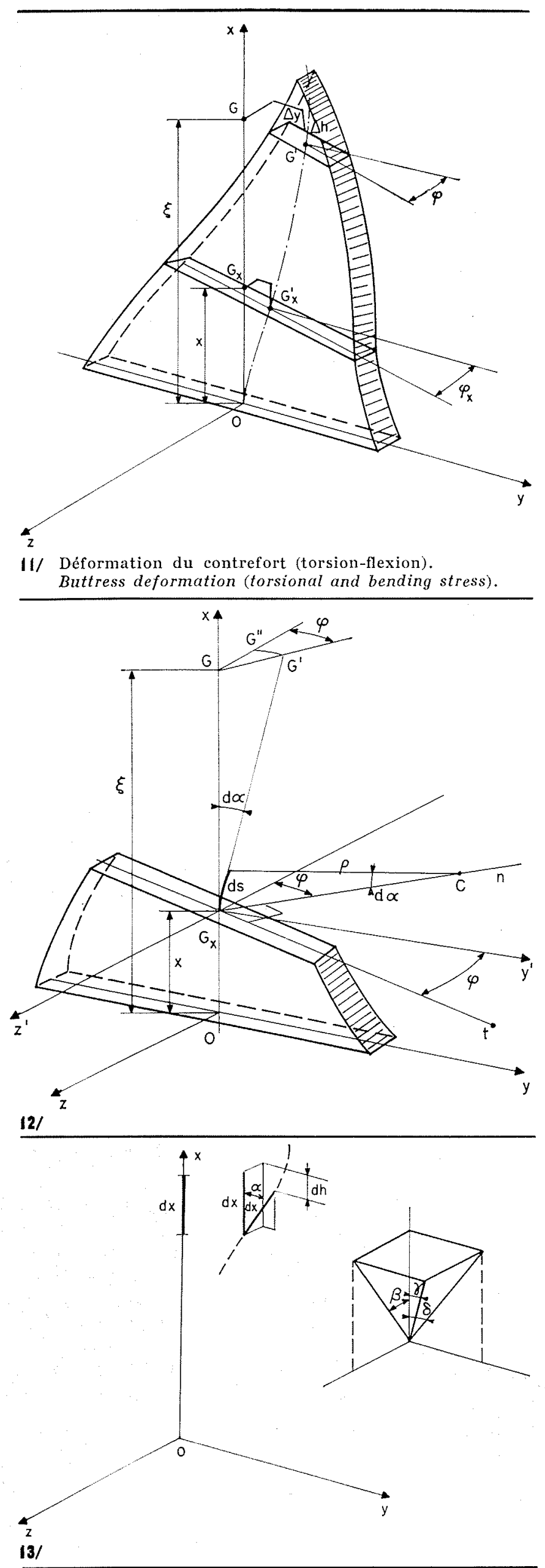

angle $\varphi$ avec le plan xoy. Cette circonstance provoque un déplacement $\Delta y$ du point $\mathrm{G}$ suivant l'axe $\mathrm{O} y$ (fig. 11).

En outre, l'incurvation de la fibre moyenne entraîne un abaissement $\Delta h$ de ce même point.

En résumé, le vecteur déplacement du point $G$ admet une composante suivant chacun des axes de coordonnées. Nous allons ci-dessous calculer successivement la composante $\Delta y$, puis la composante $\Delta h$.

- Calcul de la composante $\Delta y$ du déplacement de $G$ (fig. 12). — Nous évaluerons le déplacement infinitésimal $d y$ du point $G$, conséquence de la courbure affectant un petit élément $d s$ de la fibre moyenne situé à la cote $x$, l'angle de torsion de la section droite correspondant à cette cote étant $\varphi$.

Comme nous ne calculons pour l'instant que la contribution exclusive de la courbure de cet élément au déplacement de $G$, nous pouvons considérer que la partie inférieure du contrefort située au-dessous de la cote $x$ est simplement tordue. L'axe $\mathrm{O} x$ contient alors le centre de gravité $\mathrm{G} x$ de la section droite à la cote $x$. On peut définir ainsi un nouveau trièdre trirectangle de référence $\mathrm{G}_{x} x y^{\prime} z^{\prime}$ dans lequel $\mathrm{G}_{x} z^{\prime}$ et $\mathrm{G}_{i x} y^{\prime}$ sont respectivement parallèles à $\mathrm{O} z$ et à $\mathrm{O} y$.

$\mathrm{G}_{n} t$ étant le grand axe de symétrie de la section droite de la plaque, le centre de courbure $\mathrm{C}$ est situé sur la normale $G_{x i n} n$ à $G_{x} t$, cette normale faisant un angle $\varphi$ avec l'axe $\mathrm{G}_{x} z^{\prime}$.

Le déplacement $\overline{G G^{\prime}}$ de $G$ consécutif à la flexion du petit élément $d z$ a donc lieu dans le plan $n G_{i x} x$, faisant également l'angle $\varphi$ avec le plan $z^{\prime} \mathbf{G}_{2} x$.

On a :

$$
\mathrm{GG}^{\prime}=\mathrm{G}_{r} \mathrm{G} d \alpha
$$

avec : $d \alpha=d s / \rho$, où $\rho$ est le rayon de courbure en $\mathrm{G}_{x}$.

La composante $\overline{\mathrm{G}^{\prime \prime} \mathrm{G}^{\prime}}$ de ce déplacement suivant Oy a pour expression :

$$
\begin{gathered}
\overline{\mathrm{G}^{\prime \prime} \mathrm{G}^{\prime}}=d y=\overline{\mathrm{GG}^{\prime}} \operatorname{tg} \varphi=\overline{\mathrm{GG}^{\prime} \varphi}(\varphi \text { étant très petit) } \\
d y=\mathrm{G}_{x} \mathrm{G} \varphi \frac{d s}{\rho}=(\xi-x) \varphi \frac{d s}{\rho}
\end{gathered}
$$

En vertu de nos hypothèses, on peut écrire:

$$
\frac{d s}{\rho}=\frac{d^{2} z}{d x^{2}} d x
$$

d'où :

$$
d y=(\xi-x) \varphi \frac{d^{2} z}{d x^{2}} d x
$$

et :

$$
\Delta y=\int_{0}^{\xi}(\xi-x) \varphi \frac{d^{2} z}{d x^{2}} d x
$$

- Calcul de la composante $\Delta h_{1} d u$ déplacement de G (fig. 13). - Pour un petit élément $d x$, dont la longueur initiale, rappelons-le, demeure conservée au cours de la déformation, l'abaissement de G qui résulte de l'incurvation de la fibre moyenne a pour valeur :

$$
d h=d x(1-\cos \gamma)
$$


Entre l'angle $\gamma$ et les angles $\beta$ et $\delta$ formés respectivement par les projections sur les plans $x \mathrm{O} z$ et $x \mathrm{O} y$ de la tangente à la fibre moyenne déformée, et l'axe $O x$, on a la relation :

or :

$$
\operatorname{tg} \gamma=\sqrt{\operatorname{tg}^{2} \beta+\operatorname{tg}^{2} \delta}
$$

$$
\cos \gamma=\frac{1}{\sqrt{1+\operatorname{tg}^{2} \gamma}}=\frac{1}{\sqrt{1+\operatorname{tg}^{2} \beta+\operatorname{tg}^{2} \gamma}}
$$

soit, en développant :

$$
\cos \gamma=1-\frac{1}{2}\left(\operatorname{tg}^{2} \beta+\operatorname{tg}^{2} \gamma\right)+\ldots
$$

mais :

d'où :

$$
\operatorname{tg}^{2} \beta=\left(\frac{d z}{d x}\right)^{2} \quad \text { et } \quad \operatorname{tg}^{2} \delta=\left(\frac{d y}{d x}\right)^{2}
$$

$$
d h=\frac{1}{2}\left[\left(\frac{d z}{d x}\right)^{2}+\left(\frac{d y}{d x}\right)^{2}\right] d x
$$

L'abaissement total de $G$ a ainsi pour expression :

$$
\Delta h=\frac{1}{2} \int_{0}^{\xi}\left[\left(\frac{d z}{d x}\right)^{2}+\left(\frac{d y}{d x}\right)^{2}\right] d x
$$

$2^{\circ}$ Déplacement relatif de l'extrémité amont de la section initialement horizontale à la cote $\xi$, par rapport à des axes parallèles aux axes de référence et dont l'origine est au centre de gravité $\mathrm{G}^{\prime}$ de la section déplacée:

On a précédemment calculé les composantes du vecteur $\mathrm{GG}^{\prime}$.

Il s'agit maintenant de calculer celles du vecteur $\mathrm{M}^{\prime} \mathrm{M}^{\prime \prime}$ correspondant aux notations du croquis cidessous (fig. 14). Le déplacement total du point $M$ par rapport au système Oxyz, sera égal à la somme géométrique des vecteurs $\overline{G G}^{\prime}$ et $\bar{M}^{\prime} \mathbf{M}^{\prime \prime}$, soit :

$$
\overline{\mathrm{MM}^{\prime \prime}}=\overline{\mathrm{GG}^{\prime}}+\overline{\mathrm{M}^{\prime} \mathrm{M}^{\prime \prime}}
$$

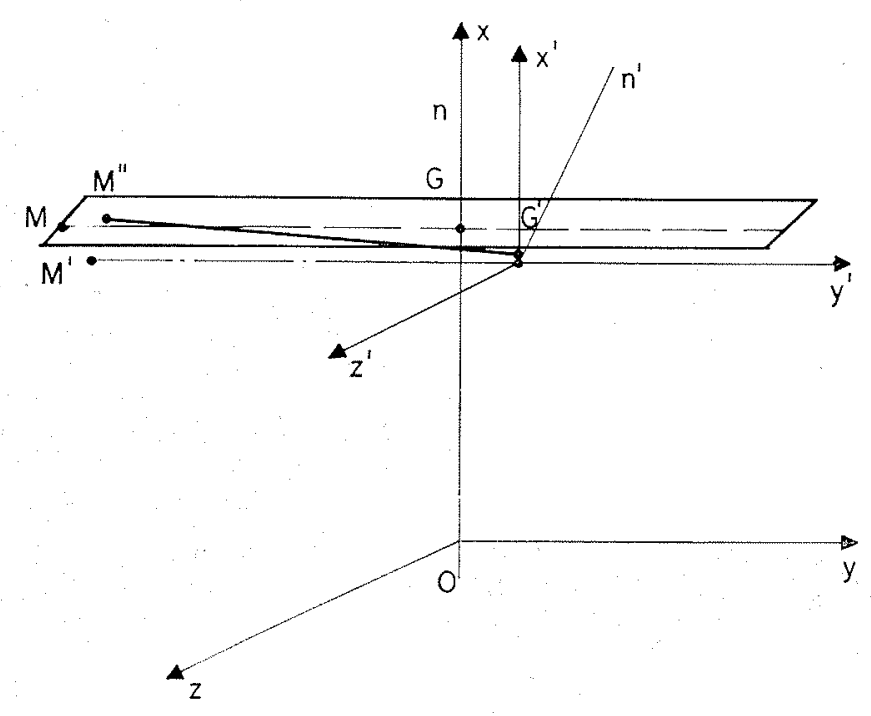

Si la normale à la section horizontale de la plaque restait parallèle à $\mathrm{O} x$, le point $\mathrm{M}$ situé à l'extrémité amont de cette section viendrait en $\mathbf{M}^{\prime}$, le vecteur $\overline{M^{\prime} M^{\prime}}$ étant équipollent au vecteur $\overline{G G^{\prime}}$.

Mais les projections de cette normale $\mathrm{G}^{\prime} n^{\prime}$ orientée suivant la tangente à la fibre moyenne déformée font respectivement :

- dans le plan $x^{\prime} G^{\prime} y^{\prime}$, l'angle $\widehat{A}_{1}$ avec l'axe $G^{\prime} x^{\prime}$, tel que :

$$
\widehat{A}_{1} \simeq \operatorname{tg} \widehat{A}_{1}=\frac{d y}{d x}
$$

- dans le plan $y^{\prime} G^{\prime} z^{\prime}$, l'angle $\varphi$ avec l'axe $G^{\prime} y^{\prime}$.

- dans le plan $x^{\prime} G^{\prime} z^{\prime}$, l'angle $\widehat{A}_{2}$ avec l'axe $G^{\prime} x^{\prime}$, tel que :

$$
\widehat{A}_{2} \approx \operatorname{tg} \widehat{A}_{2}=\frac{d z}{d x}
$$

L'équation du plan normal à $G^{\prime} n^{\prime}$, c'est-à-dire du plan qui contient $M^{\prime \prime}$, s'écrit par suite :

$$
x^{\prime}+\left(\frac{d y}{d x}\right) y^{\prime}+\left(\frac{d z}{d x}\right) z^{\prime}=0
$$

Et celle de la droite $\mathrm{G}^{\prime} \mathrm{M}^{\prime \prime}$ :

$$
\left\{\begin{array}{l}
y^{\prime}=-\frac{z^{\prime}}{\varphi} \\
x^{\prime}=z^{\prime}\left[\frac{1}{\varphi}\left(\frac{d y}{d x}\right)-\left(\frac{d z}{d x}\right)\right]
\end{array}\right.
$$

On posera $\overline{\mathrm{G}^{\prime} \mathrm{M}^{\top}}=l=\overline{\mathrm{GM}}=$ demi-longueur de la section horizontale du contrefort au niveau considéré.

On en déduit la composante suivant $\mathrm{G}^{\prime} x^{\prime} \mathrm{du}$ vecteur $\bar{M}^{\top} M^{\prime \prime}$ (toujours compté positivement lorsque le déplacement s'effectue vers le bas).

Pour $y^{\prime}=-l$, et $z^{\prime}=+l \varphi$, les équations cidessus donnent :

$$
\begin{aligned}
\Delta h_{2}=-x^{\prime}=-\left[-l\left(\frac{d y}{d x}-\frac{d z}{d x}\right)\right] & \\
& =l\left[\varphi \frac{d z}{d x}-\frac{d y}{d x}\right]
\end{aligned}
$$

La composante du vecteur $\overline{M^{\prime} M^{\prime \prime}}$ suivant $G^{\prime} y^{\prime}$ sera donnée par :

$$
\Delta y_{2}=l\left[1-\cos \left(\mathrm{G}^{\prime} \mathrm{M}^{\prime \prime}, \mathrm{G}^{\prime} y^{\prime}\right)\right]
$$

or :

$$
\cos \left(\mathrm{G}^{\prime} \mathrm{M}^{\prime \prime}, \mathrm{G}^{\prime} y^{\prime}\right)=\frac{1}{\sqrt{[d \bar{y} / d \bar{x})-\varphi(d z / d \bar{x})]^{2}+\varphi^{2}+1}}
$$

dont le développement en série permet d'écrire en négligeant les quantités de degré supérieur à 2 :

$\cos \left(\mathrm{G}^{\prime} \mathrm{M}^{\prime \prime}, \mathrm{G}^{\prime} y^{\prime}\right)=1-\frac{1}{2}\left[\varphi^{2}+\left(\frac{d y}{d x}-\varphi \frac{d z}{d x}\right)^{2}\right]$

d'où :

$$
\Delta y_{2}=\frac{l}{2}\left[\varphi^{2}+\left(\frac{d y}{d x}-\varphi \frac{d z}{d x}\right)^{2}\right]
$$


Composante $d u$ vecteur $\overline{M^{\prime} \mathrm{M}^{\prime \prime}}$ suivant $G^{\prime} z^{\prime}$ :

$$
\Delta z_{2}=l \varphi
$$

$3^{\circ}$ Abaissement complémentaire dâ $\dot{a}$ la torsion :

On sait que les sections droites d'un prisme sollicité en torsion pure se gauchissent au cours de cette torsion, sauf exclusivement si le prisme est un cylindre circulaire.

Si la longueur du prisme doit, du fait de liaisons aux extrémités, demeurer invariable, ce gauchissement donne naissance à des tensions parallèles à l'axe.

Au contraire, si comme dans le cas qui nous intéresse, il n'y a pas de liaison à une des extrémités, il n'y a pas de tensions autres que celles de cisaillement, mais le gauchissement provoque des déformations, parallèles à l'axe, des extrémités des sections droites.

Nous calculerons ci-dessous une valeur approchée de cette déformation (fig. 15 et 16).

Deux sections horizontales distantes de $d x$ tournent l'une par rapport d'un angle $\beta$ tel que :

$$
\beta=\frac{d \varphi}{d x} d x
$$

A l'extrémité de la section, du fait de la distorsion, la réduction de hauteur de la tranche d'épaisseur $d x$ est donnée par :

avec :

$$
d h_{3}=d x(1-\cos \varepsilon)
$$

$$
\begin{gathered}
\operatorname{tg} \varepsilon=\frac{\beta l}{d x}=l \frac{d \varphi}{d x} \\
\cos \varepsilon=1-\frac{\varepsilon^{2}}{2}+\ldots ; \quad d h_{3}=\frac{\varepsilon^{2}}{2} d x
\end{gathered}
$$

d'où :

$$
d h_{3}=\frac{l^{2}}{2}\left(\frac{d \varphi}{d x}\right)^{2} d x
$$

L'abaissement du point $M$ à la cote $\xi$ dû à la torsion s'écrit done :

$$
\Delta h_{3}=\frac{1}{2} \int_{0}^{\xi} l^{2}\left(\frac{d \varphi}{d x}\right)^{2} d x
$$

$4^{\circ}$ Composantes du déplacement de $\mathrm{M}$ par rapport aux axes Oxyz:

Le vecteur représentatif de ce déplacement s'écrit :

$$
\overline{\mathrm{MM}^{\prime \prime}}=\overline{\mathrm{GG}^{\prime}}+\overline{\mathrm{M}^{\prime} \mathrm{M}^{\prime \prime}}+\overline{\Delta h_{3}}
$$

en tenant compte de (23).

D'où la valeur des composantes résultant des formules établies ci-dessus :

- Suivant $\mathrm{O} x$ :

soit :

$$
\Delta h=\Delta h_{1}+\Delta h_{2}+\Delta h_{3}
$$

$$
\begin{gathered}
\Delta h=\frac{1}{2} \int_{0}^{\xi}\left[\left(\frac{d y}{d x}\right)^{2}+\left(\frac{d z}{d x}\right)^{2}\right] d x \\
+l\left[\varphi \frac{d z}{d x}-\frac{d y}{d x}\right]_{x=\xi}+\frac{1}{2} \int_{0}^{\xi} l^{2}\left(\frac{d \varphi}{d x}\right)^{2} d x \\
- \text { Suivant } \mathrm{O}_{y}: \\
\Delta y=\Delta y_{1}+\Delta y_{2}
\end{gathered}
$$

soit :

$$
\begin{aligned}
\Delta y=\int_{0}^{\xi} & (\xi-x) \varphi \frac{d^{2} z}{d x} d x+ \\
& +\frac{l}{2}\left[\varphi^{2}+\left(\frac{d y}{d x}-\varphi \frac{d z}{d x}\right)^{2}\right]_{x=\xi}
\end{aligned}
$$

- Suivant $\mathrm{O} z$ :

soit :

$$
\Delta z=\Delta z_{1}+\Delta z_{2}
$$

$$
\Delta z=[f(x)+l \varphi]_{x=\xi}
$$

D - TravaIl DEs forces appliquéEs a L'ÉlÉMENT AU COURS DE LA DÉFORMATION :

$1^{\circ}$ Poussée hydrostatique :

On a trouvé pour les composantes de la poussée élémentaire agissant à la cote $\xi$, sur la tranche d'épaisseur $d \xi$ :

$$
\begin{gathered}
d p_{v}=p_{0} \frac{f}{h}(h-\xi) d \xi \\
d p_{h}=\frac{p_{0}}{h}(h-\xi) d \xi
\end{gathered}
$$

Le travail de la force $d p_{v}$ est donné par :

$$
d \mathrm{~T}_{v}=d p_{v} \Delta h
$$

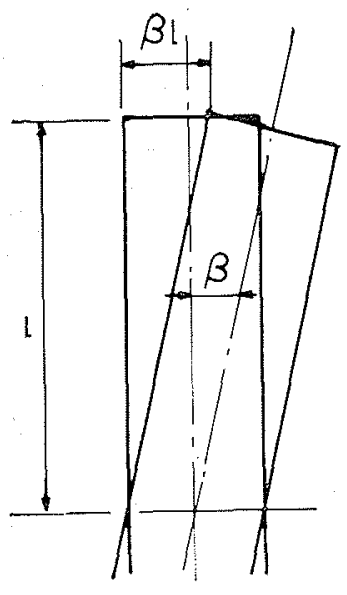

15/
15/ Rotation de la section. Rotation of the section.

16/ Déformation à l'extrémité de la section.

Deformation at the end of the section.

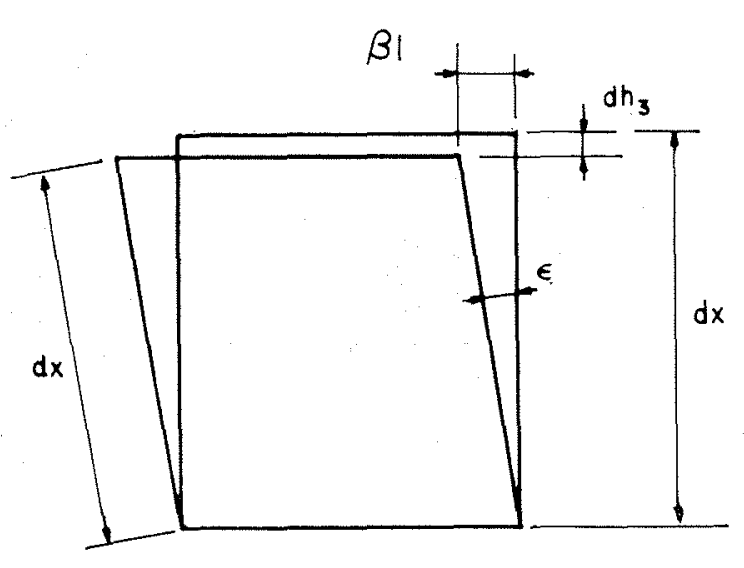

I6/ 


\section{P. PATIN et $G$. DEGEORGES}

soit :

$\mathrm{T}_{v}=p_{0} \frac{f}{h} \int_{0}^{h}(h-\xi)\left\{\frac{1}{2} \int_{0}^{\xi}\left[\left(\frac{d y}{d x}\right)^{2}+\left(\frac{d z}{d x}\right)^{2}\right] d x+l\left(\varphi \frac{d z}{d x}-\frac{d y}{d x}\right)_{x=\xi}+\frac{1}{2} \int_{0}^{\xi} l^{2}\left(\frac{d \varphi}{d x}\right)^{2} d x\right\} d \xi$

Celui de la force $d p_{\mathrm{H}}$ par :

$$
d T_{\mathrm{II}}=d p_{\mathrm{II}} \Delta y
$$

d'où : $\quad \mathrm{T}_{\mathrm{rI}}=\frac{p_{0}}{h} \int_{0}^{h}(h-\xi)\left\{\int_{0}^{\xi}(\xi-x) \varphi \frac{d z^{2}}{d x^{2}} d x+\frac{l}{2}\left[\varphi^{2}+\left(\frac{d \underline{y}}{d x}-\varphi \frac{d z}{d x}\right)^{2}\right]_{x=\xi}\right\} d \xi$

Le travail de la poussée hydrostatique est donné par la somme:

$2^{\circ}$ Travail du poids propre du contrefort:

$$
\mathrm{T}_{\mathrm{I}}=\mathrm{T}_{v}+\mathrm{T}_{\mathrm{II}}
$$

$$
d \mathrm{~T}_{2}=d \mathrm{P} \times \Delta h_{1}
$$

c'est-à-dire :

$$
\begin{aligned}
& \mathrm{T}_{2}=2 \text { epf } \int_{0}^{h} \cdot(h-\xi)\left\{\frac{1}{2} \int_{0}^{\xi}\left[\left(\frac{d y}{d x}\right)^{2}+\left(\frac{d z}{d x}\right)^{2}\right] d x\right\} d \xi \\
& \mathrm{T}_{2}=e \rho f \int_{0}^{h}(h-\xi)\left\{\int_{0}^{\xi}\left[\left(\frac{d y}{d x}\right)^{2}+\left(\frac{d z}{d x}\right)^{2}\right] d x\right\} d \xi
\end{aligned}
$$

$3^{\circ}$ Travail consécutif à la variation de courbure des voútes adjacentes :

On a trouvé :

$$
d \mathrm{~T}_{3}=p_{0} \frac{\mathrm{A}}{2 \mathrm{ch}}(h-\xi) \Delta z^{2} d \xi
$$

d'où :

$$
\mathrm{T}_{3}=p_{0} \frac{\mathrm{A}}{2 \mathrm{ch}} \int_{0}^{l_{k}}(h-\xi)[f(x)+l \varphi]^{2}{ }_{\mathrm{x}=\xi} d \xi
$$

$4^{\circ}$ Travail du couple dû à la réaction élastique du rocher le long de la base du contrefort :

L'inclinaison sur la verticale de la tangente à la fibre moyenne déformée à l'origine, est égale à la rotation de la surface de contact béton-rocher :

d'où :

$$
\begin{gathered}
\alpha=\left(\frac{d z}{d x}\right)_{x=0} \\
\mathrm{~T}_{4}=-b e^{2} \mathrm{E}_{r} \frac{m^{2}}{m^{2}-1} \frac{\pi}{36}\left(\frac{d z}{d x}\right)_{x=0}^{2}
\end{gathered}
$$

E - Variation du Potentiel interne du sXstème:

$1^{\circ}$ Variation du potentiel interne $\mathrm{U}_{1}$ des voûtes:

On fera appel à la relation (11) précédemment établie, qui s'écrit ici, compte tenu de la valeur de $\Delta z$ :

$$
\mathrm{U}_{1}=\frac{\mathrm{E}}{48 \mathrm{R}^{2}\left(\varphi \mathrm{Y}-\mathrm{X}^{2}\right) h^{3}}\left(2 \mathrm{BX}+\mathrm{RB}^{2} \mathrm{Y}+\frac{\varphi}{\mathrm{R}}\right) \int_{0}^{h}\left[e_{0}(h-\xi)+e_{h} \xi\right]^{3}[f(x)+l \varphi]^{2}{ }_{x=\xi} d \xi
$$

$2^{\circ}$ Variation de l'énergie interne $\mathrm{U}_{2}$ du contrefort :

Elle est donnée par la somme de la variation d'énergie interne $\mathrm{U}_{\mathrm{F}}$ due à la flexion et de celle $\mathrm{U}_{\mathrm{T}}$ due à la torsion.

$$
\mathrm{U}_{2}=\mathrm{U}_{\mathrm{F}}+\mathrm{U}_{\mathrm{T}}
$$

$\mathrm{M}$ et $\mathrm{T}$ étant respectivement le moment de flexion et l'effort tranchant qui sollicitent le contrefort à la cote $x$, on a :

$$
\mathrm{U}_{\mathrm{F}}=\int_{0}^{h} \frac{\mathrm{M}^{2}}{\mathrm{EI}} d x+\int_{0}^{h} \frac{\alpha \mathrm{T}^{2}}{2 \mathrm{GS}} d x
$$

I est le moment d'inertie de la section horizontale du contrefort à la cote $x$, par rapport à son axe parallèle à $\mathrm{O} y$;

$S$ est l'aire de cette section;

$\alpha$ un coefficient tenant compte de l'inégale répartition des cisaillements sur la section;

$G$ est le module de rigidité du béton au cisaillement.

On peut aussi écrire l'expression ci-dessus, puisque :

$$
\begin{gathered}
\mathrm{M}=\mathrm{EI} \frac{d^{2} z}{d x^{2}} \quad \text { et } \quad \mathrm{T}=\mathrm{EI} \frac{d^{3} z}{d x^{3}} \\
\mathrm{U}_{\mathrm{F}}=\frac{\mathrm{E}}{2} \int_{0}^{h} \mathrm{I}\left(\frac{d^{2} z}{d x^{2}}\right)^{2} d x+\frac{\alpha \mathrm{E}^{2}}{2 \mathrm{G}} \int_{0}^{h} \frac{\mathrm{I}^{2}}{\mathrm{~S}}\left(\frac{d^{3} z}{d x^{3}}\right) d x
\end{gathered}
$$


L’énergie de torsion sera calculée par la formule :

$$
\mathrm{U}_{\mathrm{T}}=\int_{0}^{h} \frac{\mathrm{C}}{2}\left(\frac{d \varphi}{d x}\right)^{2} d x
$$

dans laquelle $\mathrm{C}$ est le coefficient de rigidité de la section.

Pour une section rectangulaire, Timoshenko a montré que l'on peut écrire avec une très bonne approximation :

$$
\mathrm{C}=\frac{2 l e^{3}}{3}\left(1-0,63 \frac{e}{2 l}\right) \mathrm{G}
$$

$l$ étant, rappelons-le, la demi-longueur de la section :

D'où :

$$
\mathrm{U}_{\mathrm{T}}=\mathrm{G} e^{3} \int_{0}^{h}\left(\frac{d \varphi}{d x}\right)^{2}\left(\frac{l}{3}-0,105 e\right) d x
$$

$3^{\circ}$ Variation globale de l'énergie interne du système:

$$
\begin{aligned}
\mathrm{U}=\mathrm{U}_{1}+\mathrm{U}_{2} & =\frac{\mathrm{E}}{48 \mathrm{R}^{2}\left(\varphi \mathrm{Y}-\mathrm{X}^{2}\right) h^{3}}\left(2 \mathrm{BX}+\mathrm{RB}^{2} \mathrm{Y}+\frac{\varphi}{\mathrm{R}}\right) \int_{0}^{h}\left[e_{0}(h-\xi)+e_{h} \xi\right]^{3}[f(x)+l \varphi]^{2}{ }_{x=\xi} d \xi \\
& +\frac{\mathrm{E}}{2} \int_{0}^{h} \mathrm{I}\left(\frac{d^{2} z}{d x^{2}}\right)^{2} d x+\frac{\alpha \mathrm{E}^{2}}{2 \mathrm{G}} \int_{0}^{h} \frac{\mathrm{I}^{2}}{\mathrm{~S}}\left(\frac{d^{3} z}{d x^{3}}\right)^{2} d x+\mathrm{G} e_{3} \int_{0}^{h}\left(\frac{d \varphi}{d x}\right)^{2}\left(\frac{l}{3}-0,105 e\right) d x
\end{aligned}
$$

F - Choix des fonctions de déformation $z=f(x)$ ET $\varphi=\varphi(x)$ :

$1^{\circ}$ Fonction $z=f(x)$ :

Elle doit satisfaire aux conditions aux extrémités suivantes :

- au sommet du contrefort, où le moment fléchissant et l'effort tranchant sont l'un et l'autre nuls :

- au niveau de la surface d'appui :

$$
\frac{d^{2} z}{d x^{2}}=0 \quad \text { et } \quad \frac{d^{3} z}{d x^{3}}=0
$$

Le long de cette surface, puisque l'on a convenu de négliger la déformation tangentielle du rocher, on devra avoir simultanément :

$$
x=0 \quad \text { et } \quad z=0
$$

En outre, on peut faire apparaître une relation entre les dérivées seconde et première, c'est-à-dire entre l'inclinaison de la tangente à l'origine et le moment de flexion en ce point.

En effet, nous avons :

$$
\begin{gathered}
\mathrm{M}=b e^{2} \mathrm{E}_{r} \frac{m^{2}}{m^{2}-1} \frac{\pi}{18} \alpha \\
\alpha=\left(\frac{d z}{d x}\right)_{x=0} \quad \text { et } \quad \mathrm{M}=+\left(\mathrm{EI} \frac{d z^{2}}{d x^{2}}\right)_{p=0}
\end{gathered}
$$

(compte tenu de nos conventions de signe)

d'où :

$$
\left(\mathrm{EI} \frac{d^{2} z}{d x^{2}}\right)_{x=0}=\left(b e^{2} \mathrm{E}_{r} \frac{m^{2}}{m^{2}-1} \frac{\pi}{18} \frac{d z}{d x}\right)_{x=0} \text { avec } \mathrm{I}=\frac{b e^{3}}{12}
$$

ou :

$$
{\frac{d^{2} z}{d x^{2}}}_{(x=0)}=\frac{3 \pi}{4 e} \frac{\mathrm{E}_{r}}{\mathrm{E}}{\frac{m^{2}}{m^{2}-1}}_{\frac{d z}{d x}}(x=0)
$$

On posera :

$$
\lambda=\frac{3 \pi}{4 e} \frac{\mathrm{E}_{r}}{\mathrm{E}} \frac{m^{2}}{m^{2}-1}
$$

et l'on obtient :

$$
{\frac{d^{2} z}{d x^{2}}}_{(x=0)}=\lambda \frac{d z}{d x}_{(x=0)}
$$

Il est plus aisé de satisfaire aux conditions ci-dessus en choisissant pour $f(x)$ un développement en série de fonctions algébriques.

La fonction la plus simple qui convienne est la suivante :

$$
z=a\left[x+\frac{\lambda}{2} x^{2}-\frac{\lambda x^{3}}{3 h}+\frac{\lambda}{12 h^{2}} x^{4}\right]
$$

Mais elle n'est pas suffisante pour représenter le phénomène de voilement avec assez de généralité. En fait, si nous avions décidé, lors de l'étude de la première partie, de prendre en compte l'influence de la flexion transversale du contrefort, c'est cette fonction que nous aurions dû retenir.

On peut facilement montrer qu'elle traduit simplement la flexion transversale corrélative à l'existence du moment le long de la base, à l'exclusion de toute autre cause.

En effet, pour que ce moment soit nul, il suffit d'admettre que le coefficient d'élasticité du rocher est nul, ce 
qui revient à poser $k=0$. L'équation (36) prend alors la forme simplifiée :

$$
z=\alpha x
$$

que nous avons précédemment utilisée.

Il est donc nécessaire d'ajouter un second paramètre qui traduira la flexion transversale du contrefort résultant des phénomènes de voilement.

La fonction la plus simple qui convienne s'écrit alors :

$$
\begin{aligned}
z=a_{1}\left(x+\frac{\lambda x^{2}}{2}-\right. & \left.\frac{\lambda}{4 h^{2}} x^{4}+\frac{\lambda}{10 h^{3}} x^{5}\right) \\
& +a_{2}\left(x^{3}-\frac{x^{4}}{h}+\frac{3 x^{5}}{10 h^{2}}\right)
\end{aligned}
$$

Nous nous contenterons dans ce qui suit de cette fonction.

Il serait bien entendu possible de la compléter en y ajoutant d'autres paramètres. Mais les calculs qui en résulteraient deviendraient très vite à peu près inextricables.

\section{$2^{\circ}$ Fonction $\varphi=\varphi(x)$ :}

On doit avoir :

- à la base :

$\varphi=0$ (puisqu'on néglige la déformation tangentielle du rocher);

- au sommet :

$$
\frac{d \varphi}{d x}=0 \text { (absence de couple) }
$$

Il est avantageusement possible de satisfaire simultanément à ces deux conditions en choisissant la fonction trigonométrique :

$$
\varphi=b \sin \frac{\pi x}{2 h}
$$

qui peut être regardée comme le premier terme du développement en série de Fourier de la fonction $\varphi$ exacte.

\section{TROISIEME PARTIE}

\section{Application numérique}

\section{A - Définition sommatre de L'ouvrage examiné.}

Les caractéristiques essentielles du barrage sont indiquées sur le croquis (fig. $17 a$ et $17 b$ ).

Il s'agit d'un ouvrage particulièrement mince, plus élancé que les grands barrages à voûtes multiples réalisés à ce jour (du moins à notre connaissance).

Approximativement, les contraintes maximales de compression dans les voûtes sont de l'ordre de $100 \mathrm{~kg} / \mathrm{cm}^{2}$. Dans le contrefort, au point le plus sollicité, la contrainte de compression atteint $70 \mathrm{~kg} / \mathrm{cm}^{2}$ environ.

Nous ne préciserons pas la définition exacte des parements des voûtes et nous nous bornerons seulement à dire que les arcs horizontaux qui en résultent sont circulaires et d'épaisseur constante à un niveau donné, la variation d'épaisseur de la voûte étant une fonction linéaire de la cote $x$.

Par simplification, le fruit des génératrices de l'extrados des voûtes sera pris égal à celui du parement amont du contrefort, soit 0,60 .

- densité du béton : $\rho=2,40$.

- coefficient d'élasticité du béton :

$$
\mathrm{E}=1,50 \times 10^{6} \frac{\mathrm{T}}{\mathrm{m}^{2}}
$$

Ce coefficient est pris assez faible pour tenir compte implicitement des déformations différées dues au fluage sous charge du béton.

Module de rigidité du béton au cisaillement :

$$
\mathrm{G}=7 \times 10^{5} \frac{\mathrm{T}}{\mathrm{m}^{2}}
$$

Coefficient de section : $\alpha=1,50$ (rectangle).

Coefficient d'élasticité du rocher d'appui :

$$
\mathrm{E}_{r}=1,50 \times 10^{6} \frac{\mathrm{T}}{\mathrm{m}^{2}}
$$

Coefficient de Poisson de ce rocher : $\mu=0,20$ d'où :

$$
m=\frac{1}{\mu}=5
$$

\section{a) élévation - elevation}

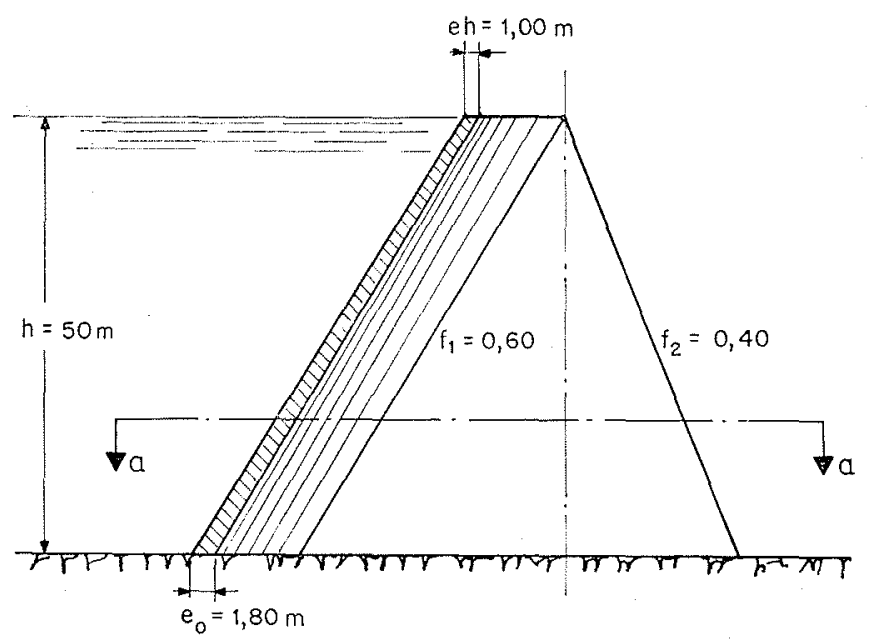

b) coupe-section a-a

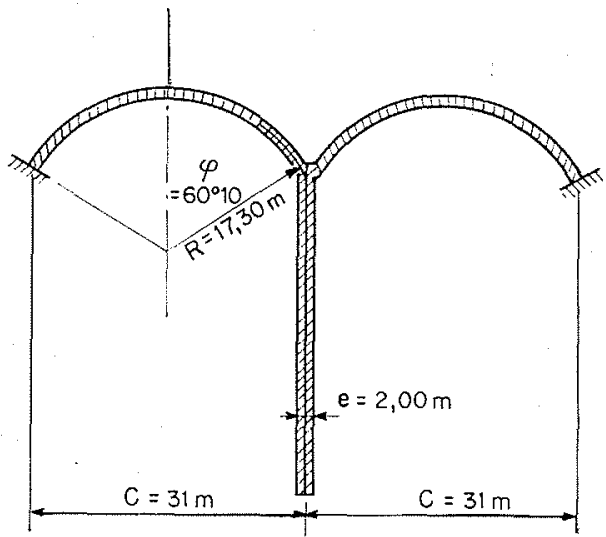

17/ 
B - Stabilité D'ensemble au déversement.

Les quantités $\beta, \gamma, \delta$ et $\zeta$ ont respectivement les valeurs suivantes :

- calcul de $\beta$ :

$$
\beta=\frac{f_{1} h^{3} c}{12}=2 \times 10^{5}
$$

- calcul de $\gamma$ :

$$
\gamma=\frac{\mathrm{A} h^{4}}{24}=10,70 \times 10^{5}
$$

avec :

$$
A=\frac{\cos \varphi+\varphi \sin \varphi}{\sin \varphi-\varphi \cos \varphi}=4,092
$$

- calcul de $\delta$ :

$$
\begin{gathered}
\delta=\frac{\rho\left(f_{1}+f_{2}\right) e h^{3}}{12}-\varepsilon b e^{2} \mathrm{E}_{r} \frac{m^{2}}{m^{2}-1} \cdot \frac{\pi}{36} \\
\frac{\rho\left(f_{1}+f_{2}\right) e h^{3}}{12}=0,50 \times 10^{5}
\end{gathered}
$$

Le calcul du second terme de $\delta$, représentatif de la participation du rocher d'appui, donne pour valeur :

$$
\varepsilon b e^{2} \mathrm{E}_{r} \frac{m^{2}}{m^{2}-1} \cdot \frac{\pi}{36}=272 \times 10^{5} \varepsilon
$$

On obtiendra une valeur vraisemblable du coefficient $\varepsilon$ en considérant l'équation (36) qui permet de calculer la rotation réelle au niveau de la fondation lors d'un déversement.

$$
z=\alpha\left(x+\frac{\lambda x^{2}}{2}-\frac{\lambda x^{3}}{3 h}+\frac{\lambda x^{4}}{12 h^{2}}\right)
$$

La rotation est donnée par le coefficient $\alpha$. Le déplacement en tête, c'est-à-dire pour $x=h$, obtenu par cette équation, est égal à :

$$
z=\alpha h\left(\frac{1+\lambda h}{4}\right)
$$

Alors que la forme linéaire adoptée donne : $z=a h$.

Le rapport entre le coefficient angulaire $a$ et la rotation $\alpha$ a donc pour valeur :

$$
\alpha\left(\frac{1+h}{4}\right)=a h
$$

soit :

$$
\alpha=\frac{a}{1+(\lambda h / 4)}
$$

L'angle de rotation intervenant au carré dans l'expression du travail $\mathrm{T}_{4}$, on aura :

$$
\varepsilon=\frac{1}{[1+(\lambda h / 4)]^{2}}=\left(\frac{\alpha}{a}\right)^{2}
$$

$\lambda=\frac{3 \pi}{4 e} \frac{\mathrm{E}_{r}}{\mathrm{E}} \frac{m^{2}}{m^{2}-1}=4,22$ d'où $\varepsilon=3,85 \times 10^{-3}$

Finalement :

$$
\varepsilon b e^{2} \mathrm{E}_{r} \frac{m^{2}}{m^{2}-1} \frac{\pi}{36}=1,04 \times 10^{5}
$$

et :

$$
\delta=-0,54 \times 10^{5}
$$

Les calculs numériques ci-dessus montrent la grande influence de la flexion propre du contrefort, sur la grandeur de la réaction du rocher. Cette flexion qui s'effectue autour de la déformée linéaire considérée, n'affecte par contre que fort peu le travail des forces latérales agissant sur le contrefort. - calcul de $\zeta$ :

$$
\begin{aligned}
\zeta=\frac{\mathrm{Eh}^{3}\left[2 \mathrm{BX}+\mathrm{RB}^{2} \mathrm{Y}+(\varphi / \mathrm{R})\right]}{48 \mathrm{R}^{2}\left(\varphi \mathrm{Y}-\mathrm{X}^{2}\right)} \\
\quad \times\left(\frac{e_{0}^{3}}{30}+\frac{e_{0}^{2} e_{h}}{10}+\frac{e_{0} e_{h}^{2}}{5}+\frac{e_{h}^{3}}{3}\right)
\end{aligned}
$$

où :

$$
\begin{aligned}
& \mathrm{B}=\frac{\varphi}{2 \mathrm{R}(\varphi \cos \varphi-\sin \varphi)}=-0,0881 ; \\
& \mathrm{X}=\sin \varphi-\varphi \cos \varphi=0,3441
\end{aligned}
$$

et :

$$
\begin{gathered}
\mathrm{Y}=\frac{\varphi}{2}+\varphi \cos ^{2} \varphi-\frac{3}{4} \sin 2 \varphi=0,1370 \\
\frac{2 \mathrm{BX}+\mathrm{RB}^{2} \mathrm{Y}+(\varphi / \mathrm{R})}{\varphi \mathrm{Y}-\mathrm{X}^{2}}=0,727 \\
\zeta=114 \times 10^{5}
\end{gathered}
$$

$1^{\circ}$ Cas d'un contrefort unique associé aux deux voûtes adjacentes:

On trouve:

$$
k=\frac{\zeta-\delta}{\beta+\gamma}=9,00
$$

$2^{\circ}$ Barrage constitué par deux contreforts et trois voûtes :

Le déterminant (17) se réduit à :

$\left|\begin{array}{ll}2[k(\beta+\gamma)+\delta-\zeta] & \zeta-k \gamma \\ \zeta-k \gamma & 2[k(\beta+\gamma)+\delta-\zeta]\end{array}\right|=0$

dont les racines s'écrivent respectivement :

soit :

$$
k_{1}=\frac{\zeta-2 \delta}{2 \beta+\gamma} ; \quad k_{2}=\frac{3 \zeta-2 \delta}{2 \beta+3 \gamma}
$$

$$
k_{1}=7,83 \quad ; \quad k_{2}=9,50
$$

La première racine, la plus petite, est celle que l'on doit retenir.

$3^{\circ}$ Barrage constitué par trois contreforts et quatre voutes:

On a, au cours de l'exposé, calculé les racines correspondant à ce cas et qui ont pour valeurs respectives :

$k_{1}=\frac{\zeta-\delta}{\beta+\gamma} \quad ; \quad k_{2}=\frac{\zeta(\sqrt{2}-1)-\sqrt{2} \delta}{\sqrt{2} \beta+(\sqrt{2}-1) \gamma}$

$$
k_{3}=\frac{\zeta(\sqrt{2}+1)-\sqrt{2} \delta}{\sqrt{2} \beta+(\sqrt{2}+1) \gamma}
$$


soit numériquement :

$$
k_{1}=9,00 \quad k_{2}=6,60 \quad k_{3}=9,75
$$

le coefficient de sécurité correspond à la deuxième racine.

\section{Cas de contreforts plus nombreux:}

Nous indiquerons ci-dessus la plus petite racine de l'équation obtenue, en développant le déterminant (17) pour les valeurs successives suivantes du nombre de contreforts :

$$
\begin{array}{ll}
\text { pour } n=4 ; & k=5,80 \\
\text { pour } n=5 ; & k=5,00 \\
\text { pour } n=6 ; & k=4,70 \\
\text { pour } n=7 ; & k=4,55 \\
\text { pour } n=9 ; & k=4,30
\end{array}
$$

Les chiffres qui précèdent montrent qu'à partir d'une certain nombre de contreforts, le coefficient de sécurité au déversement ne décroît plus que fort lentement, son taux de variation n'étant surtout appréciable que pour les tout premiers contreforts. Sauf, peut-être, si la suite $k$ ne converge pas et lorsque le barrage aura une grande Iongueur, on pourra généralement négliger cette variation audelà de $n=10$.

Les résultats précédents montrent le peu d'intérêt qui s'attache à la création de points fixes intermédiaires le long du tracé d'un barrage à voûtes multiples de grande longueur.

Ces points fixes, obtenus par des culées poids, à moins qu'ils ne soient très proches l'un de l'autre (trois ou quatre éléments de barrage), ne conféreront qu'un gain relativement modeste au coefficient de sécurité au déversement. Par contre, les culées poids sont des ouvrages massifs, - calculés pour absorber la poussée totale agissant sur un seul de leurs côtés, — dont l'existence augmentera de façon sensible le coût du barrage.

Pour améliorer le coefficient de sécurité, il sera préférable d'agir sur les paramètres entrant dans l'expression donnant $k$.

On peut montrer que l'on y parviendra en rapprochant les contreforts, en réduisant l'angle au centre des arcs et bien entendu en augmentant l'épaisseur des voûtes.

On risque toutefois d'aboutir à une mauvaise utilisation du béton entrant dans la confection de ces voûtes.

Sans entrer dans le détail, nous dirons simplement que, les contraintes maximales gardant toujours la même valeur, on ne pourra augmenter la stabilité au déversement qu'en acceptant du même coup un accroissement des quantités de béton entrant dans la construction du barrage.

\section{C - Stabilité élastigue d’un élément constitué PAR UN CONTREFORT ET LES DEUX VOÛTES ADJACENTES :}

\section{Remarque :}

On a posé, dans l'établissement des formules que nous allons utiliser, que les faces latérales du contrefort étaient des triangles isocèles. Nous ramènerons le problème étudié à ce cas en faisant choix

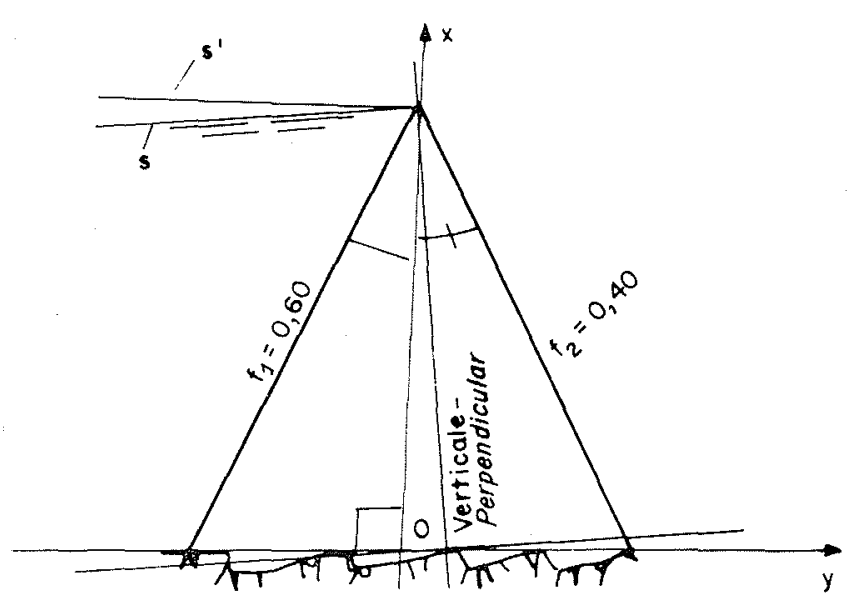

18/

d'un axe $\mathrm{O} x$ confondu avec la bissectrice de l'angle au sommet du contrefort.

Une telle assimilation ne peut être admise que parce que l'axe $O x$ ainsi défini est voisin de la perpendiculaire à la base.

Néanmoins, cette base étant en réalité assez rarement horizontale, on peut toujours supposer que son inclinaison est telle que cette condition d'orthogonalité soit satisfaite.

On est ainsi conduit à imaginer par cet artifice une base inclinée vers l'aval (fig. 18).

Cette disposition n'est pas incompatible avec la stabilité au glissement, puisqu'il est possible de créer le long de la base une succession de redans convenablement orientés.

Par rapport au nouvel axe $O x$, les fruits des deux parements sont égaux et l'on a sensiblement: $f=0,50$.

Appliquer nos formules, revient à prendre $S^{\prime}$ comme surface libre de la retenue au lieu de $S$.

L'approximation ainsi commise ne peut avoir qu'une faible influence sur le résultat et conduit à une sous-évaluation de la charge critique, donc à un résultat pessimiste en ce qui concerne le coefficient de sécurité calculé.

Nous avons précédemment parlé des fonctions de déformation $f$ et $\varphi$ définissant respectivement la flexion et la torsion du contrefort.

Nous ne donnerons pas ici le détail des calculs numériques (d'ailleurs assez longs) nécessaires au calcul de la charge critique.

Nous avons obtenu un coefficient de sécurité $k=2,15$ chiffre beaucoup plus faible que celui trouvé pour la seule stabilité au déversement.

Il n'en sera pas toujours ainsi. En effet, les caractéristiques propres du contrefort n'ont que fort peu d'influence sur le déversement en bloc, alors que son voilement dépend étroitement de ses dimensions et des qualités du béton qui le constitue. Nous avons dit au début de la présente étude que pour la plupart des barrages à voûtes multiples actuellement en service, les contraintes dans les contreforts sont assez modestes, contrairement à celles qui sollicitent les voûtes.

Il nous paraît très probable que, si l'on appliquait à ces ouvrages les calculs de la présente note, nous aboutirions à une conclusion différente, le 
déversement en bloc étant alors plus à craindre que le voilement des contreforts.

\section{Conclusions.}

Les résultats auxquels nous sommes parvenus semblent toutefois montrer que la stabilité des barrages à voûtes multiples sera toujours convenablement assurée pour des ouvrages d'une hardiesse encore supérieure à la plupart de ceux qui ont été récemment édifiés.

Si l'on s'en tient au principe des calculs exposés, on remarquera que toutes les hypothèses émises sont pessimistes. On serait donc en droit de s'attendre à des coefficients de sécurité supérieurs à ceux que nous avons obtenus pour les divers cas examinés s'il était possible de prendre en compte de façon plus exacte les conditions de la réalité.

Cependant, du point de vue strictement mathématique, on ne peut espérer avoir choisi la vraie valeur des fonctions $f$ et $\varphi$, et là on ne peut pécher que par défaut.

On pourrait bien sûr serrer davantage la vérité en prenant des termes complémentaires des développements en série de ces fonctions.

Rappelons toutefois que nous nous sommes seulement proposé de fixer des ordres de grandeur. Nous ne pensons pas d'ailleurs que l'on puisse demander davantage à nos calculs dans lesquels certaines conditions de compatibilité, en particulier dans l'évaluation des déformations, ne peuvent être vérifiées.

A notre avis, si une solution plus rigoureuse de ce problème devait être recherchée, elle devrait s'appuyer sur des fondements théoriques plus complets.

Nous espérons reprendre ultérieurement ce travail en nous efforçant de réduire le nombre des simplifications admises ici, notamment en substituant la théorie des plaques à celle des poutres.

\section{Extension de la méthode au cas des barrages à contreforts dotés d'un écran d'étanchéité rigide}

Les calculs précédemment exposés peuvent, bien entendu, s'appliquer aux ouvrages dont la partie amont est constituée par une dalle massive inclinée vers l'aval et qui prend appui sur un contrefort triangulaire. Des définitions nombreuses et variées ont été données, dans les barrages existants, à cette dalle, mais, d'une façon très générale, ces barrages sont caractérisés par un rapport $e / c$ de l'épaisseur $e$ des contreforts à leur espacement $c$ supérieur à celui des voûtes multiples. Ce fait s'explique aisément par les conditions de sollicitation de la matière constitutive des têtes des éléments travaillant partiellement en console, qui limitent très vite cet écartement (fig. 19 a et 19 b).

De la sorte, les contraintes maximales dans les contreforts deviennent en pratique toujours inférieures à celles que l'on observe dans les contreforts de barrages à voûtes multiples. C'est pour-

quoi, en général, sauf peut-être pour d'exceptionnelles hauteurs d'ouvrages, le danger de voilement ne paraît guère devoir être envisagé dans ce cas.

Cependant, la transposition de la méthode de calcul aux barrages à contreforts et dalle amont épaisse, soulève quelques difficultés pour préciser les conditions auxquelles est assujettie la lisière amont du contrefort.

Du point de vue constructif, on réalise souvent un joint imbriqué entre la dalle et le contrefort. Seul, en définitiye, le frottement consécutif à l'effort de compression le long de ce joint, s'oppose, lors d'un voilement de contrefort, à un déplacement relatif de la lisière amont du contrefort par rapport à la dalle, qui, pour sa part, peut être regardée transversalement, c'est-à-dire dans le sens du barrage, comme infiniment rigide. Si le frottement est suffisant, tout se passe comme si la lisière amont se trouvait partiellement encastrée dans la dalle. Au contraire, si localement l'amorce d'un voilement du contrefort conduit à un effort tranchant en ce point supérieur au frottement, un glissement relatif pourrait survenir dans le plan du joint.

$\mathrm{Vu}$ sous cet aspect, le problème semble des plus ardus. C'est pourquoi, nous admettrons un tel glissement impossible. En fait, pour qu'un tel déversement se réalise, il devrait se produire sur l'ensemble du barrage, les éléments de l'écran d'étanchéité se butant les uns les autres sans possibilité, comme dans les voûtes, de flexion élastique. Un tel mouvement supposerait donc le déplacement d'une culée qui ne subit normalement aucune poussée. C'est donc un accident hautement improbable.

Par contre, dans l'étude du voilement, notre méthode étant par ailleurs impuissante à traduire l'influence, favorable à la stabilité élastique du contrefort, de son encastrement partiel dans la dalle, - elle suppose indéformables les sections horizontales du contrefort - nous serons, à cet égard, conduits à sous-évaluer la charge critique.

Si nous désirions prendre en compte cet encastrement, nous devrions alors faire appel à la théorie des plaques, la déformation en un point quelconque du contrefort ne pouvant être dans ce cas exprimée en fonction de la seule variable $x$.

Nos hypothèses conduisent à exprimer les conditions au pourtour de la façon suivante :

- lisière inférieure : élastiquement appuyée sur le rocher;

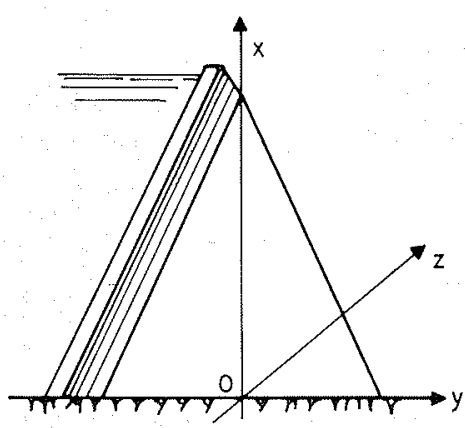

a)

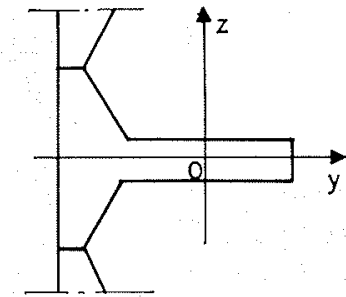

b)

$19 /$ 
- lisière aval : bord libre;

- lisière amont: composante du déplacement nulle suivant $\mathrm{OZ}$.

En outre, le sommet du contrefort appartenant également à la lisière amont, le déplacement suivant $O Z$ de ce point sera constamment égal à 0 .

Si nous reprenons, pour représenter la flexion latérale de la fibre moyenne, la fonction précédemment envisagée dans notre application numérique, c'est-à-dire :

$$
\begin{aligned}
f(x)=a_{1}\left(x+\frac{\lambda x^{2}}{2}-\frac{\lambda x^{4}}{4 h}\right. & \left.+\frac{\lambda}{10 h^{3}} x^{5}\right) \\
& \quad+a_{2}\left(x^{3}-\frac{x^{4}}{h}+\frac{3 x^{5}}{10 h^{2}}\right)
\end{aligned}
$$

cette dernière condition implique que : $f(h)=0$, d'où :

$$
a_{2}=-\frac{a_{1}}{3 h^{2}}(10+17 k h)
$$

et :

$$
f(x)=a_{1}\left[\begin{array}{l}
x-\frac{x^{3}}{3 h^{2}}+\frac{x^{4}}{3 h^{3}}-\frac{x^{5}}{h^{4}} \\
+\lambda\left(\frac{x^{2}}{2}-\frac{x^{4}}{4 h^{2}}+\frac{x^{5}}{10 h^{3}}\right)
\end{array}\right]
$$

La composante du déplacement latéral le long de la lisière amont s'exprime, comme on l'a vu précédemment, en fonction du déplacement latéral $f(x)$ de la fibre moyenne et de l'angle de torsion $\varphi$, par :

$$
\Delta z=f(x)+\varphi l \quad \text { avec } \quad l=(h-x) f
$$

Sur la lisière amont on aura donc :

$$
f(x)+(h-x) f \varphi=0
$$

d'où :

$$
\varphi=\frac{a_{1}}{(h-x) f}\left[\begin{array}{l}
-x+\frac{x^{3}}{3 h^{2}}-\frac{x^{4}}{3 h^{3}}+\frac{x^{\overline{5}}}{h^{4}} \\
-\lambda\left(\frac{x^{2}}{2}-\frac{x^{4}}{4 h^{2}}+\frac{x^{5}}{10 h^{3}}\right)
\end{array}\right]
$$

Ainsi, avec la fondation choisie, la déformation latérale du contrefort, compte tenu des conditions aux limites, s'exprime en définitive avec un paramètre unique : $a_{1}$.

On peut cependant améliorer l'approximation du calcul en complétant la fonction $f(x)$ par un terme supplémentaire comportant un second paramètre si on le désire.

Le calcul de l'énergie de déformation propre du contrefort ne pose en lui-même aucune difficulté formelle. Par contre, celui du travail de la poussée hydrostatique présente ici une indétermination nouvelle qui n'existait pas dans le cas des voûtes multiples.

En effet, nous n'avons écrit nulle condition quant à la déformation de la lisière amont, si ce n'est que son déplacement latéral était nul.

Rien ne s'oppose donc à un mode de voilement tel

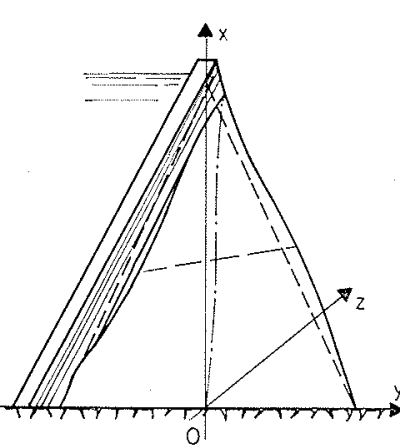

a) $e^{e r} \cos -$ Case I

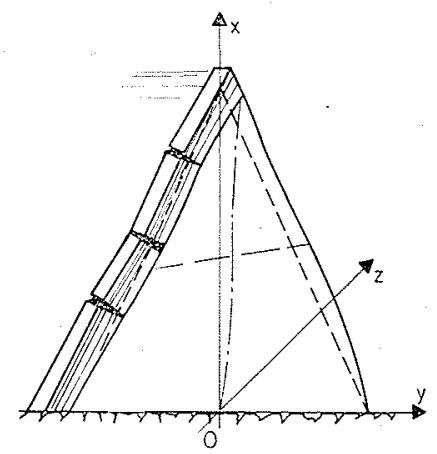

b) $2^{\text {ime cas }-C a s e ~} 2$
$20 /$

que la rectitude de cette lisière soit altérée par la déformation. Si cette rectitude n'était pas conservée, deux cas seraient alors à envisager.

- ou la dalle est suffisamment résistante pour pallier localement l'absence de support du contrefort en mobilisant sa propre rigidité en flexion (fig. $20 a$ );

- ou la dalle n'est pas apte à résister à cet effort de flexion. Dans ce dernier cas, la rupture qui résulte de cette insuffisance conduit à rétablir le contact entre la dalle et le contrefort (fig. $20 \mathrm{~b}$ ).

Laquelle de ces deux hypothèses convient-il de retenir?

Si l'on s'en tient à la première, le déplacement de la dalle consistera en une simple rotation autour de sa base déterminée par le déplacement suivant OY du sommet du contrefort. Le calcul du travail de la poussée hydrostatique est alors immédiat.

Si l'on admet au contraire la seconde, tout se passe à peu près, la dalle étant rompue, comme sì la poussée hydrostatique était directement appliquée à la lisière amont du contrefort. La valeur du travail de l'eau sera alors plus grande que celle trouvée dans le cas précédent.

Par contre, il serait nécessaire de considérer comme nous l'avons fait pour les voûtes dans l'étude précédente, la variation du potentiel total de l'élément de barrage à contrefort, à savoir :

- variation du potentiel interne $d u$ contrefort (flexion latérale et torsion);

- variation du potentiel interne de la dalle (flexion).

Outre que, dans le deuxième cas, l'évaluation de l'énergie de flexion de la dalle est analytiquement assez difficile, nous pensons que l'on peut tout aussi valablement se contenter de la première hypothèse.

En fait, avec les types actuels de barrages à contreforts, un tel problème peut difficilement se poser : le contrefort se développe en champignon pour constituer la dalle et l'ensemble est extrêmement rigide : vu sous cet angle, c'est une poutre à section en $\mathrm{T}$ et la lisière amont du contrefort ne peut se déformer seule. Il n'est donc en pratique 
qu'un seul mode de déformation possible, celui étudié précédemment.

\section{Conclusion.}

Nous n'avons pas cherché, dans cette étude, à épuiser le sujet du flambement et du déversement des contreforts : des ouvrages entiers seraient nécessaires pour tenir compte de tous les cas possibles. Nous avons essayé de limiter ce nombre de cas en faisant des hypothèses simplificatrices, toujours orientées dans le sens de la sécurité. Nous sommes ainsi certains de pouvoir nous montrer plus hardis et par suite plus économes sans franchir des limites de sécurité encore très larges. C'est à cela, croyons-nous, que doit tendre le progrès dans l'art de l'ingénieur.
S. Timosmroro, -- Théorie de la stabilité élastique (Béranger).

F. Vogr. - Über die Berechnung der Fundament Deformation (Osio, 1925).

A. Passaro. - Considerazioni sull'instabilita dell'equilibrio elastico delle travi a mensola con profilo triangolare (l'Energia Elettrica, février 1957).

Symetrical buckling of Right. - Angled Isocele triangular plates, par WITTRICK (department of aeronautical engineering, University of Sydney).

Buckling of a triangular plate by shearing forces, par IhuTCHIOFF (Belgrade).

Pour le flambement des arcs: Sur la stabilité de l'équilibre des voûtes circulaires soumises à une pression uniforme, par E. Baticle (Le Génie Civil, mai 1960).

\section{Abstract \\ Stability problems associated with buttress dams, and more especially dams of the multiple-arch type * \\ by P. Patin ** and G. Degeorges **}

Concrete working stresses remain low in most buttress dams because designers are anxious to avoid buckling and lateral overturning effects at all cost. By precise examination of these two forms of failure, safety factors can be determined for this type of structure.

Multiple-arch structures, for which it seems more difficult to achieve stable design than for other buttress structures, are fortunately fairly easily designed. Their case is discussed first in the article.

OVERALL LATERAL OVERTURNING STABILITY OF A MULTIPLE-ARCH DAM

The following assumptions are made in defining the considered structure:-

(i) All arches and buttresses identical, also in height;

(ii) Triangular buttress side faces, and uniform buttress thickness;

(iii) Arches in the form of elliptical cylinders with circular horizontal cross-sections of constant thickness. Arch thickness variation linear from the dam crest to its foot.

\section{A) Other assumptions.}

1. Regarding hydrostatic thrust distribution.

The entire hydrostatic thrust is assumed to be acting on the arches, and either through them or directly on to the upstream buttress faces. In other words, the part of this thrust absorbed by the arch foundations is neglected.

\section{Regarding buttress supports.}

The buttresses are considered to be standing on the rock (unconstrained) and the concrete-to-rock contact surface is considered as an unilateral connection only capable of transmitting compressive stress.

* The first part of this paper has been published in La Houille Blanche, n 7-1965, pp. 681-690.

* Directeur Général de la Compagnie d'Ingénieurs et Techniciens d'Etudes, Paris.

** Ingénieur-Conseil à la Compagnie d'Ingénieurs et Techniciens d'Etudes, Paris. 
The rock is assumed to be elastic with homogeneous and isotropic properties. Use is made of the data Vogt found, starting out from the conventional Boussinesq formulae.

Only relationships between moment and rotation are considered, i.e. the invariably slight additional deformation due to shear is neglected.

3. Regarding the arches.

These are considered as stacks of horizontal arcs incapable of mutual shear stress transmission in their separation planes.

\section{Regarding approximate displacement magnitude.}

Displacement magnitude is determined from a certain number of known parameters $a_{1}, a_{2}, \ldots$ These are assumed to always be such small quantities that terms in which they are of a higher degree than the second can reasonably be neglected.

\section{B) Basic theoretical data.}

The calculations discussed in the following are based on the Lejeune-Dirichlet equilibrium stability theorem, which states that when the forces applied to a material system and the internal forces of that system derive from a total potential $\mathrm{V}$, the stable equilibrium positions for the system are those for which $\mathrm{V}$ is a minimum.

Here, the internal forces involved will be the internal stresses in the structure, which are known to derive from a potential U. The applied forces (water thrust and ground reaction, the former resulting from gravity, and the latter an elastic force) also derive from a potential, $\mathrm{U}^{\prime}$, this being equal to the total work done by the applied forces, but of opposite sign: $\mathrm{U}^{\prime}=-\mathrm{T}$ :

$$
\mathrm{V}=\mathrm{U}^{\prime}+\mathrm{U}^{\prime}=\mathrm{U}-\mathrm{T}
$$

Stable equilibrium positions will be those for which variation of $\mathrm{V}$ is zero:

$$
\delta(\mathrm{U}-\mathrm{T})=0
$$

\section{C) Lateral overturning stability of a system consisting of a buttress and its two adjacent arches.}

The buttress is assumed to rotate about its base through an angle " $a$ " and the work done by the applied forces and the variation in internal potential of the system are both calculated.

1. WORK DONE BY APPLIED FORCES.

a) Water thrust:

Let $p_{0}$ be the critical unit load acting at the foot of the buttress and causing it to overturn. If $p_{r}$ is the true load, the safety factor will be given by:

$$
k=\frac{p_{0}}{p_{r}}
$$

If $f_{1}=\tan V_{1}$ is the upstream face batter, work due to thrust of the water can be expressed by:

Eq. (3)

b) Buttress weight:

If $p$ is the specific gravity of the concrete and $f_{2}=\tan V_{2}$ is the downstream buttress batter, work due to buttress weight is given by:

Eq. (4)

c) Lateral thrust due to adjacent buttress curvature variation:

As the buttress upsets, the curvature of one of the adjacent arches increases while that of the other decreases.

Arch radius variation is expressed by:

$$
\Delta \mathrm{R}=-\frac{\mathrm{S}}{\varphi^{2}} \Delta \varphi
$$

where $\varphi$ is half the angular opening of each arch, $\mathrm{R}$ is the arch radius at level $x$, and $\mathrm{S}$ is half the arch length.

This variation is expressed in terms of chord variation $\Delta z$.

If :

$$
\mathbf{A}=\frac{\cos \varphi+\varphi \sin \varphi}{\sin \varphi-\varphi \cos \varphi}
$$

the final relationship for the work done by the arches is: 
d) Work done by rock reaction along the buttress bearing face:

Vogt has shown rotation $\alpha$ of the concrete-to-rock contact face and moment $M$ due to rock reaction to be related as follows:-

$$
\mathrm{M}=b e^{2} \alpha \mathrm{E}_{r} \frac{m^{2}}{m^{2}-1} \frac{\pi}{18}
$$

where $E_{r}$. is the Iongitudinal elasticity coefficient for the rock and $m=(1 / \mu)$ is the reciprocal of Poisson's ratio.

Work done by the couple thus determined is then given by:

$$
\mathrm{T}_{4}=b e^{2} \mathrm{E}_{r} \frac{m^{2}}{m^{2}-1} \frac{\pi}{36} a^{2}
$$

The actual amount of rotation is attenuated by lateral flexion of the buttress due (precisely) to the reaction couple, however, so that:

in which $\varepsilon$ is a numerical coefficient smaller than 1, the value of which will be determined in the numerical application.

e) Work resulting from the applied forces:

This is equal to the following sum:

$$
\mathrm{T}=\mathrm{T}_{1}+\mathrm{T}_{2}+\mathrm{T}_{3}+\mathrm{T}_{4}
$$

\section{Internal SyStem potential variation.}

As the buttress is assumed incapable of buckling in this case, lateral overturning solely affects internal arch energy. Considered only as affected by the thrust of the water, this internal energy has a certain value $U_{1}$, and as the distance between the supports closes in or opens out, internal energy changes to $U_{2}$. The quantity to be determined is the variation $\mathrm{U}=\mathrm{U}_{2}-\mathrm{U}_{1}$.

If $\mathrm{C}$ is the couple applied at the abutments of each arch and $\mathrm{Q}$ is the thrust component along the chord, Castigliano's theorem can be written as follows:-

$$
\frac{\delta \mathrm{U}}{\delta \mathrm{C}}=\int_{0}^{s} \frac{\mathrm{M}}{\mathrm{EI}} \frac{\delta \mathrm{M}}{\delta \mathrm{C}} d s=-\Delta \varphi ; \quad \frac{\delta \mathrm{U}}{\delta \mathrm{Q}}=\int_{0}^{s} \frac{\mathrm{M}}{\mathrm{EI}} \frac{\delta \mathrm{M}}{\delta \mathrm{Q}} d s=\Delta z
$$

and by writing:

$$
\mathrm{B}=\frac{\varphi}{2 \mathrm{R}(\varphi \cos \varphi-\sin \varphi)} ; \quad \sin \varphi-\varphi \cos \varphi=\mathrm{X} ; \quad \frac{\varphi}{2}+\varphi \cos ^{2} \varphi-\frac{3}{4} \sin 2 \varphi=\mathrm{Y}
$$

one obtains the following linear system:

$$
\mathrm{C} \varphi+\mathrm{QRX}=-\mathrm{B} \frac{\mathrm{EI} \Delta z}{2 \mathrm{R}} ; \quad \mathrm{CX}+\mathrm{QRY}=\frac{\mathrm{EI} \Delta z}{2 \mathrm{R}^{2}}
$$

from which the following can be calculated:

$$
\mathrm{G}=-\frac{\mathrm{EI}}{2 \mathrm{R}^{2}} \frac{\mathrm{X}+\mathrm{RBY}}{\mathrm{Y}-\mathrm{X}^{2}} \Delta z ; \quad \mathrm{Q}=\frac{\mathrm{EI}}{2 \mathrm{R}^{3}} \frac{\mathrm{RBX}+\varphi}{\mathrm{Y}-\mathrm{X}^{2}} \Delta z
$$

Clapeyron's formulae give internal energy variation as:

$$
\mathrm{U}=\frac{\mathrm{C}(-\Delta \varphi)}{2}+\frac{\mathrm{Q} \Delta z}{2}
$$

Hence, if $e_{0}$ is the arch thickness at its base and $e_{h}$ that at its crest, final calculation yields the following relationship:

$$
\text { Eq. (13) }
$$

\section{EQUILIBRIUM CONDITION.}

Here, the relationship $\delta(\mathrm{U}-\mathrm{T})=0$ reduces to $\mathrm{U}-\mathrm{T}=0$, so that, from the above calculations:

$$
\text { Eq. (14) }
$$

and hence the safety factor is given by $h=p_{0} / p_{r}=p_{0} / h c$

With the following: Eq. (15)

equation (14) comes out as $k(\beta+\gamma)+\delta=\zeta$

Whence

$$
k=\frac{\zeta-\delta}{\beta-\gamma}
$$




\section{P. PATIN et G. DEgeorges}

D) Overall lateral overturning stability of a dam consisting of three buttresses and four identical arches.

The outermost arches are considered to be rigidly anchored. The considered system is completely defined in any position by the values of parameters $a_{1}, a_{2}$ and $a_{3}$, which, for each buttress, are similar to the parameter $a$ previously defined.

\section{Calculation of $\mathrm{T}$.}

Because of the mutual independence of parameters $a_{1}, a_{2}$ and $a_{3}$ and buttress similarity, the following are obtained:

$$
\begin{aligned}
& \mathrm{T}_{1}=p_{0} \frac{f_{1} h^{2}}{12}\left(a_{1}^{2}+a_{2}^{2}+a_{3}{ }^{2}\right) \\
& \mathrm{T}_{2}=p \frac{\left(f_{1}+f_{2}\right) e h^{3}}{12}\left(a_{1}^{2}+a_{2}^{2}+a_{3}^{2}\right) \\
& \mathrm{T}_{4}=-\varepsilon b e^{2} \mathrm{E}_{r} \cdot \frac{m^{2}}{m^{2}-1} \frac{\pi}{36}\left(a_{1}{ }^{2}+a_{2}{ }^{2}+a_{3}{ }^{2}\right)
\end{aligned}
$$

Calculation of $\mathrm{T}_{3}$ :

This calculation only differs from the previous one in that the chord variations for the different arches are not the same. Final calculation yields the following:

$$
\mathrm{T}_{3}=p_{0} \frac{\mathrm{A} h^{3}}{24 \mathrm{C}}\left(a_{1}^{2}+a_{2}^{2}+a_{3}^{2}-a_{1} a_{2}-a_{2} a_{3}\right)
$$

2. Calculation of U.

Here too, the internal energy variations are different for each arch. By exactly the same reasoning as above, $\mathrm{U}$ is seen to be given by the following expression:

$$
\mathrm{U}=\frac{\mathrm{Eh}^{3}\left[2 \mathrm{BX}+\mathrm{RB}^{2} \mathrm{Y}+(\varphi / \mathrm{R})\right]}{48 \mathrm{R}^{2}\left(\varphi \mathrm{Y}-\mathrm{X}^{2}\right)}\left(\frac{e_{0}{ }^{3}}{30}+\frac{e_{0}{ }^{2} e_{h}}{10}+\frac{e_{0} e_{h}^{2}}{5}+\frac{e_{h}{ }^{3}}{3}\right)\left(a_{1}{ }^{2}+a_{2}{ }^{2}+a_{3}{ }^{2}-a_{1} a_{2}-a_{2} a_{3}\right)
$$

and for the $(\mathrm{U}-\mathrm{T})$ value:

$$
\begin{aligned}
(\mathrm{U}-\mathrm{T})= & -k \beta\left(a_{1}^{2}+a_{2}^{2}+a_{3}^{2}\right)-k \gamma\left(a_{1}^{2}+a_{2}^{2}+a_{3}^{2}-a_{1} a_{2}-a_{2} a_{3}\right) \\
& +\zeta\left(a_{1}^{2}+a_{2}^{2}+a_{3}^{2}-a_{1} a_{2}-a_{2} a_{3}\right)-\delta\left(a_{1}^{2}+a_{2}^{2}+a_{3}^{2}\right)
\end{aligned}
$$

Minimum conditions are expressed by the following:

$$
\begin{aligned}
& \frac{\delta(\mathrm{U}-\mathrm{T})}{\delta a_{1}}=2 a_{1}(k \beta+\delta)-(\zeta-k \gamma)\left(2 a_{1}-a_{2}\right)=0 \\
& \frac{\delta(\mathrm{U}-\mathrm{T})}{\delta a_{2}}=2 a_{2}(k \beta+\delta)-(\zeta-k \gamma)\left(2 a_{2}-a_{1}-a_{3}\right)=0 \\
& \frac{\delta(\mathrm{U}-\mathrm{T})}{\delta a_{3}}=2 a_{3}(k \beta+\delta)-(\zeta-k \gamma)\left(2 a_{3}-a_{2}\right)=0
\end{aligned}
$$

Rearrangement of the above gives a homogeneous linear system, the determinant of which must be zero to satisfy the compatibility condition:

$$
\left|\begin{array}{lll}
2[k(\beta+\gamma)+\delta-\zeta] & (\zeta-k \gamma) & 0 \\
(\zeta-k \gamma) & 2[k(\beta+\gamma)+\delta-\zeta] & (\zeta-k \gamma) \\
0 & (\zeta-k \gamma) & 2[k(\beta+\gamma)+\delta-\zeta]
\end{array}\right|=0
$$

Expansion yields the following equation:

$$
8[k(\beta+\gamma)+\delta-\zeta]^{3}-4[k(\beta+\gamma)+\delta-\zeta][\zeta-k \gamma]^{2}=0
$$

which is an equation of the third degree for $k$, the roots of which are easily calculated and whose respective values are:

$$
k_{1}=\frac{\zeta-\delta}{\beta+\gamma} \quad ; \quad k_{2}=\frac{\zeta(\sqrt{2}+1)-\sqrt{2} \delta}{\sqrt{2} \beta+(\sqrt{2}-1) \gamma} \quad ; \quad k_{3}=\frac{\zeta(\sqrt{2}+1)-\sqrt{2} \delta}{\sqrt{2} \beta+(\sqrt{2}+1) \gamma}
$$

The smallest of these roots is the required safety factor. 


\section{E) Overall lateral overturning stability of a dam consisting of $n$ buttresses and $n+1$ identical arches.}

This is a system with $n$ degrees of liberty, and the previous calculations are easily generalised. It can be shown by an extension of the reasoning applied above that the compatibility determinant is of the following general form:

$$
\text { Eq. (17) }
$$

This determinant has $n$ lines and $n$ columns, and the solution of the equation obtained by expanding it will yield $n$ roots, the smallest of which is the only one to be considered.

The following recurrence law is obtained from the form of the determinant $n$ :

$$
\Delta_{n}=2[k(\beta+\gamma)+\delta-\zeta] \Delta_{n-1}-\left(\zeta-k_{\gamma}\right)^{2} \Delta_{n-2}
$$

which may facilitate identification of the successive roots.

\section{F) Case of a dam with buttresses of different heights.}

All considerations so far were based on the assumption of a dam with identical buttresses, which in fact practically never applies. The calculations could easily be extended to apply to a structure with varying buttresses, however, by calculating the particular values of quantities $\beta, \gamma, \delta$ and $\zeta$ applicable to each buttress.

The more general case will now be examined in which lateral overturning is accompanied by buckling of the buttress. The implicit assumption will be made that the arches' own elastic stability remains unaffected during the considered deformations.

\section{A) Assumptions.}

In addition to the assumptions made in Part I it will be assumed for present purposes that plate forming the buttress is an isosceles triangle whose equal sides are the upstream and downstream buttress faces.

How the buttress deforms outside the plane represented by its mean plane when not under load will be considered to result from the superimposition of a torsion $\varphi=\varphi(x)$ about the axis of symmetry-i.e. about the vertical axis - and a lateral flexion along the same axis defined by the function $z=f(x)$. Although this choice may seem arbitrary, it nevertheless seems to cater for the various possible deformation modes.

\section{B) Basic theoretical data.}

The energy method will again be applied here, but first, the analytical form of the above characteristic buckling functions $\varphi$ and $f$ must be found; this will be done by Ritz's method of serial expansion of the $\varphi$ and $f$ functions of the form:

$$
z=a_{0}+a_{1} f_{1}+a_{2} f_{2}+a_{3} f_{3}+\ldots \quad ; \quad \varphi=b_{0}+b_{1} \varphi_{1}+b_{2} \varphi_{2}+b_{3} \varphi_{3}+\ldots
$$

and $(\mathrm{U}-\mathrm{T})$ can be written as follows:

$$
(\mathrm{U}-\mathrm{T})=\mathrm{F}\left(a_{0}, a_{1}, a_{2} \ldots ; b_{0}, b_{1}, b_{2} \ldots\right)
$$

and

$$
\delta(\mathrm{U}-\mathrm{T})=\frac{\delta \mathrm{F}}{\delta a_{0}} d a_{0}+\frac{\delta \mathrm{F}}{\delta a_{1}} d a_{1}+\ldots \frac{\delta \mathrm{F}}{\delta b_{0}} d b_{0}+\frac{\delta \mathrm{F}}{\delta b_{1}} d b_{1}+\ldots=0
$$

Increments $d a_{0}, d a_{1} \ldots d b_{0}, d b_{1} \ldots$ being arbitrary, the following should simultaneously hold good:

$$
\frac{\delta \mathrm{F}}{\delta a_{0}}=0 ; \frac{\delta \mathrm{F}}{\delta a_{1}}=0 ; \ldots \quad \frac{\delta \mathrm{F}}{\delta b_{0}}=0 ; \frac{\delta \mathrm{F}}{\delta b_{1}}=0 ; \ldots
$$

The resulting calculation process will thus be similar to the one applied in Part $I$.

\section{C) Calculation of displacement components for a point on the upstream buttress face.}

1. Displacement of a point G of the mean fibre with respect to trihedron Oxyz.

The displacement vector for point $G$ has a component along each coordinate axis. The one along $O z$ is given by $z=f(x)$. Components $\Delta y$ and $\Delta h$ will now each be calculated in turn. 
Calculation of $G$ displacement component $\Delta y$ :

The infinitesimal displacement $d y$ of point $G$ due to curvature affecting a small mean fibre element $d s$ at level $x$ is given by:

Eq. (18)

where $\varphi$ is the torsional angle of the cross-section at level $x$.

Calculation of $G$ displacement component $\Delta h_{1}$ :

For a small element $d x$ the initial length of which remains the same throughout the deformation process, the lowering of the position of $G$ resulting from the curvature of the mean fibre is given by

$$
d h=d x(1-\cos \gamma)
$$

Hence:

$$
d h=\frac{1}{2}\left[\left(\frac{d z}{d x}\right)^{2}+\left(\frac{d y}{d x}\right)^{2}\right] d x
$$

Which gives the following expression for the total lowering of the position of $G$ :

$$
\text { Eq. (19) }
$$

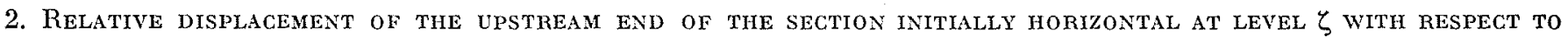
AXES PARALIel to THE REFERENCE AXES, AND THE ORIGIN OF WHICH LIES AT THE CENTRE OF GRAVITY G' OF THE DISPLACED SECTION:

If $l$ is the half-length of the horizontal buttress section at the considered level, the following is obtained:

$$
\text { Eq. (20), Eq. (21) and Eq. (22) }
$$

3. Additional lowering due to torsion,

The lowering of the position of point $M$ at level $\zeta$ due to torsion is expressed by:

$$
\text { Eq. (23) }
$$

4. Components of the uisplacement of M with respect to axes Oxyz.

The following values are found from the above calculations for displacement components for $\mathrm{M}$ :

$$
\begin{aligned}
& \text { Along Ox: } \quad \Delta h=\Delta h_{1}+\Delta h_{2}+\Delta h_{3}, \quad \text { i.e. } \quad \text { Eq. (24) } \\
& \text { Along } \mathrm{O} y: \quad \Delta y=\Delta y_{1}+\Delta y_{2}, \quad \text { i.e. } \quad \text { Eq. (25) } \\
& \text { Along } \mathrm{O} z \text { : } \Delta z=\Delta z_{1}+\Delta z_{2} \text {, i.e. Eq. (26) }
\end{aligned}
$$

D) Work done by forces applied during the deformation process.

1. Hydrostatic thrust.

The following values are obtained for the vertical and horizontal hydrostatic thrust components:

$$
\text { Eq. (27) and Eq. (28) }
$$

The work done by the hydrostatic thrust is given by the following sum:

$$
\mathrm{T}_{1}=\mathrm{T}_{v}+\mathrm{T}_{\mathrm{H}}
$$

2. WORK DONE BY THE WEIGHT OF THE BUTtRESS.

Eq. (29)

3. Work done as a result of the variation in CURvature of the adjacent arches.

Eq. $(30)$

4. WORK DONE BY THE COUPLE RESUlting FROM ELASTIC ROCK REACTION ALONG THE BUtTRESS BASE.

Eq. (31)

E) System internal potential variation.

1. Arch internal potential $U_{1}$ variation. 
2. Buttress internal energy $\mathrm{U}_{2}$ variation.

This is given by the sum of the variation of internal energy due to flexion $\mathrm{U}_{\mathrm{F}}$ and that due to torsion $\mathrm{U}_{\mathrm{T}}$ :

Hence:

$$
\mathrm{U}_{2}=\mathrm{U}_{\mathrm{F}}+\mathrm{U}_{\mathrm{T}}
$$

and by Timoshenko's formulae:

Eq. (33)

Eq. (34)

3. Overall system internal energy variation.

Eq. (35)

F) Choice of deformation functions $z=f(x)$ and $\varphi=\varphi(x)$.

1. Function $z=f(x)$.

Allowing for limit conditions, it is shown that the simplest suitable function is the following:

Eq. (37)

where:

$$
\lambda=\frac{3 \pi}{4 e} \cdot \frac{\mathrm{E}_{r}}{\mathrm{E}} \frac{m^{2}}{m^{2}-1}
$$

2. Function $\varphi=\varphi(x)$.

The limit conditions can be satisfied with the following function:

$$
\varphi=b \sin \frac{\pi x}{2 h}
$$

which can be considered as the first term in a Fourier series analysis of the exact $\varphi$ function.

PART III

NUMERICAL APPLICATION

\section{A) Brief description of the considered structure.}

The numerical application is for a particularly thin structure in which the maximum compression stresses amount to $100 \mathrm{~kg} / \mathrm{cm}^{2}$ in the arches and $70 \mathrm{~kg} / \mathrm{cm}^{2}$ in the buttresses. Constants are as follows:

Specific gravity of concrete: $\rho=2.40$;

Elasticity coefficient for concrete $\mathrm{E}=1.50 .10^{6}$ tons/sq.m;

Modulus of rigidity for concrete: $\mathrm{G}=7.10^{5}$ tons/sq.m;

Area coefficient : $\alpha=1.50$ (rectangle);

Bearing rock elasticity coefficient: $\mathrm{E}_{r}=1.50 .10^{6}$ tons $/ \mathrm{sq} \cdot \mathrm{m}$;

Poisson ratio for bearing rock: $\mu=0.20$, and hence: $m=(1 / \mu)=5$.

\section{B) Overall lateral overturning stability.}

Values of $\beta, \gamma, \delta$ and $\zeta$ are as follows:

$$
\beta=2.10^{5} \quad \gamma=10.7 .10^{5}, \quad \text { for } A=4.092
$$

To find $\delta$ it is necessary to calculate coefficient $\varepsilon$ in the moment applied to the buttress by the rock. Calculation of the true rotation shows that this coefficient is given by:

and hence:

$$
(\alpha / a)^{2}=3.85 \cdot 10^{-3}
$$

$$
\delta=-0.54 .10^{5} \quad \zeta=114.10^{5}
$$

1. Case of a single buttress with two adjacent arches.

$$
k=\frac{\zeta-\delta}{\beta+\gamma}=9.0
$$


2. DAM CONSISTING of Two BUTtResses AND THREE ARChes.

The determinant reduces to:

the roots of which are:

$$
\left|\begin{array}{ll}
2[k(\beta+\gamma)+\delta-\zeta] & \zeta-k \gamma \\
\zeta-k \gamma & 2[k(\beta+\gamma)+\delta-\zeta]
\end{array}\right|=0
$$

$$
k_{1}=7.83 \quad k_{2}=9.50
$$

The first of these roots-the smaller-is the one to be considered.

3. DaM Consisting of three buttresses and FOUR ARChEs.

The respective values of the roots for this case were calculated as follows:

$$
k_{1}=\frac{\zeta-\delta}{\beta+\gamma} \quad ; \quad k_{2}=\frac{\zeta(\sqrt{2}-1)-\sqrt{2} \delta}{\sqrt{2} \beta+(\sqrt{2}-1) \gamma} \quad ; \quad k_{3}=\frac{\zeta(\sqrt{2}-1)-\sqrt{2} \delta}{\sqrt{2} \beta+(\sqrt{2}-1) \gamma}
$$

i.e. in numerical terms:

$$
k_{1}=9.0 \quad k_{2}=6.6 \quad k_{3}=9.75
$$

The safety factor is the second of these roots.

4. More thaN three butTrfesses.

The following are the smallest roots of the equation obtained for different numbers of buttresses:

$$
\begin{aligned}
& n=4: k=5.8 \\
& n=5: k=5.0 \\
& n=6: k=4.7 \\
& n=7: k=4.55 \\
& n=8: k=4.3
\end{aligned}
$$

These figures show that lateral overturning safety factor only decreases very slowly above a certain number of buttresses.

C) Elastic stability of a buttress with its two adjacent arches.

The safety factor found was $k=2.15$, which is much less than only for lateral overturning stability. This is not always so, however, for the inherent buttress characteristics only affect its lateral overturning, whereas buckling of the buttress closely depends on its dimensions and the quality of its concrete.

\section{Conclusions}

The results of this study seem to show that satisfactory stability can be ensured for even more daring multiplearch structure designs than most of those erected to date.

PART IV

\section{EXTENSION OF THE METHOD TO THE CASE OF BUTTRESS DAMS FEATURING A RIGID GROUT CURTAIN}

Though the above calculations can of course also be applied to structures with an upstream portion in the form of a massive slab inclined towards downstream and abutting against a triangular buttress, some difficulties nevertheless arise. Examination shows failure by lateral overturning to be practically impossible, and that one needs only reckion with the buttress buckling under very definite conditions, in view of the rigidity of the upstream part of the structure.

\section{Conclusion}

With a few invariably pessimistic simplifying assumptions, an attempt was made to determine the safety coefficients for various types of buttress dam failure with a view to producing cheaper designs for these structures. 